\title{
MiR-93-5p regulates tumorigenesis and tumor immunity by targeting PD-L1/CCND1 in breast cancer
}

\author{
Meng Yang ${ }^{1,2 \#}$, Ran Xiao ${ }^{1 \#}$, Xinru Wang ${ }^{1}$, Youyi Xiong ${ }^{1}$, Zhenfeng Duan ${ }^{1,3}$, Duolu Li $^{1}$, Quancheng Kan ${ }^{1}$ \\ ${ }^{1}$ Department of Pharmacy, the First Affiliated Hospital of Zhengzhou University, Zhengzhou, China; ${ }^{2}$ Department of Academy of Medical Sciences, \\ Zhengzhou University, Zhengzhou, China; ${ }^{3}$ Sarcoma Biology Laboratory, UCLA Orthopedic Surgery, Los Angeles, CA, USA \\ Contributions: (I) Conception and design: D Li, M Yang; (II) Administrative support: Q Kan, D Li, Z Duan; (III) Provision of study materials or \\ patients: Y Xiong, X Wang; (IV) Collection and assembly of data: M Yang, R Xiao; (V) Data analysis and interpretation: M Yang; (VI) Manuscript \\ writing: All authors; (VII) Final approval of manuscript: All authors. \\ \#These authors contributed equally to this work as co-first authors. \\ Correspondence to: Quancheng Kan; Duolu Li; Zhengfeng Duan. Department of Pharmacy, the First Affiliated Hospital of Zhengzhou University, \\ Zhengzhou, China. Email: kanquancheng2021@163.com; dorali1979@126.com; zxd221@med.miami.edu.
}

Background: Challenges in medical care posed by rapid tumor progression, individualized responses to therapy, and the heterogeneous characteristics of breast cancer (BRCA) highlight the urgent need for new treatment strategies, as well as therapeutic and prognostic markers. Accumulating evidence has revealed that microRNAs broadly participate in carcinogenesis, but our understanding of the role of $m i R-93-5 p$ in BRCA remains limited.

Methods: The prognosis of miR-93-5p, programmed cell death-ligand 1 (PD-L1) and CCND1 were analyzed by datasets. Freshly excised breast cancer tissues $(\mathrm{N}=33)$ and adjacent noncancerous tissues $(\mathrm{N}=18)$ were collected to detect the expression of CCND1 and PD-L1 by immunohistochemistry (IHC). Quantitative real-time PCR (qRT-PCR) and Western blot were used to test the expression of miR-93-5p, PD-L1 and CCND1 after transfected mimics or inhibitors. Dual-luciferase reporter assay indicates the direct targeting between miR-93-5p and PD-L1.

Results: Bioinformatics analysis demonstrated that $m i R-93-5 p$ plays differential roles in various tumors, and further verification using qRT-PCR revealed that the expression levels of $m i R-93-5 p$ were lower in MDA-MB-231 cells than in noncancerous breast cells. In addition, we confirmed that PD-L1 and CCND1 generated mutual effects, and $m i R-93-5 p$ directly targets the PD-L1/CCND1 signaling pathway to influence their accumulation and distribution in the cell membrane, nucleus, and cytoplasm, mediating tumor progression and immune regulation in BRCA.

Conclusions: Taken together, $m i R-93-5 p$ could regulate tumorigenesis and tumor immunity by targeting PD-L1/CCND1 in BRCA and our research provides a rationale for therapy with $m i R-93-5 p$ to overcome immune escape and improve risk stratification.

Keywords: miR-93-5p; CCND1; programmed cell death-ligand 1 (PD-L1); immunoregulation; breast cancer (BRCA)

Submitted Dec 17, 2021. Accepted for publication Feb 21, 2022.

doi: 10.21037/atm-22-97

View this article at: https://dx.doi.org/10.21037/atm-22-97

\section{Introduction}

Breast cancer (BRCA), the second most commonly diagnosed cancer, is the leading cause of morbidity and mortality among female malignancies worldwide $(1,2)$.
Approximately $70-80 \%$ of patients with early-stage, nonmetastatic BRCA achieve good treatment outcomes, but patients who experience advanced BRCA and distant organ metastases are generally incurable using currently 
available therapies. The heterogeneity of BRCA further complicates clinical (2). Current treatment strategies for BRCA are increasingly emphasizing individualization and de-escalation/escalation according to tumor molecular subtype and early therapeutic response (2,3). Moreover, to reduce the incidence of BRCA, researchers worldwide are attempting to establish risk assessment models for BRCA and to improve the accuracy of these models (3).

MicroRNAs (miRNAs) are a class of small noncoding RNAs 19-25 nucleotides ( 22 nt) in length that are involved in posttranscriptional regulation, including translational blockade and/or messenger RNA (mRNA) degradation, by directly binding to the 3 '-untranslated regions (3'-UTRs) of target mRNAs, leading to a decrease in target protein expression $(4,5)$. To date, tremendous progress has been made in the field of miRNAs; for example, their functions have been correlated with the tumor microenvironment, epithelial-mesenchymal transition, cancer stemness, epigenetic regulation, invasive processes, exosome-mediated communication in invasion, cell adhesion, cytoskeletal structure, cell-cell adhesion junctions, and cell-extracellular matrix molecules (ECM) interactions in BRCA. Briefly, miRNAs, which act as gene regulators, participate in multiple biological processes, including cell proliferation, differentiation, apoptosis, migration, and invasion, affecting many diseases, particularly cancer (6). Many studies have indicated that miRNAs are abnormally expressed in various tumor types, acting as tumor suppressor genes or oncogenes $(4,7)$. Since 2011 , miRNAs have been recognized as ubiquitous players in the regulation of all cancer hallmarks, and targeting miRNAs as cancer therapy has attracted increasing attention $(8,9)$. Indeed, several miRNAs as therapeutic molecules have currently entered phase I clinical trials, such as $m i R-34 a, m i R-124, m i R-424, m i R-138$, and $m i R-155$. These miRNAs have a similar feature in that they all regulate immune signaling by inducing the expression of programmed cell death 1 (PD-1), Programmed cell death 1 ligand 1 (PD-L1), and cytotoxic T-lymphocyte associated protein 4 (CTLA-4) in and attracting $\mathrm{CD}^{+} \mathrm{T}$ cells to the tumor immune microenvironment (TIM) $(8,10-14)$. Generally, tumor infiltrating immune cells (innate and adaptive immunity) can effectively control tumor progression and eventually eradicate tumor cells, but tumor cells develop the ability to escape immunosurveillance and destruction through immunoediting. Hence, it is crucial to understand the role of miRNAs in controlling cancer immunity $(8,15,16)$.

Currently, there are many studies on BRCA, but there are not many reports on $m i R-93-5 p$ in BRCA. Although there have been studies on epithelial-mesenchymal transition (EMT), the target is different from that of our research group, and the immune checkpoint PD-L1, which is in the early stage of clinical application, is selected as the target in our research group. And in combination with another oncoprotein, CCND1, which has not previously been reported in BRCA. In this study, we focused on miR-93-5p, which is significantly downregulated in MDA-MB-231 BRCA cells. Our results illustrated that miR-93-5p not only inhibited cell proliferation and cell cycle progression but also attenuated migration and invasion of BRCA cells. Subsequently, we demonstrated that $m i R-93-5 p$ directly targets the PD-L1/CCND1 signaling pathway and impedes the mutual effect between PD-L1 and CCND1. In addition, bioinformatics analysis and tissue microarray analysis indicated that $m i R-93-5 p$ participates in immunoregulation. In summary, our study implicates miR-93$5 p$ as a potential immune regulatory target in the control of BRCA tumorigenesis.

We present the following article in accordance with the MDAR reporting checklist (available at https://atm. amegroups.com/article/view/10.21037/atm-22-97/rc).

\section{Methods}

\section{Bioinformatics analysis}

Kaplan-Meier Plotter was used to analyze the prognostic value of $m i R-93-5 p, \mathrm{PD}-\mathrm{L} 1$, and CCND1 in various cancers (17). TargetScan 7.2 and miWALK3.0 were used to predict the target genes of miR-93-5p using default parameters $(18,19)$. Bioinformatics \& Evolutionary Genomics (http://bioinformatics.psb.ugent.be/webtools/ Venn/) was used to intersect the two datasets. Metascape was used for gene-set enrichment analysis (20). UALCAN was used to analyze expression levels of $P D-L 1$ and CCND1 according to sample type and BRCA subtype (21). The Encyclopedia of RNA Interactomes (ENCORI) was used to analyze the correlation of $m i R-93-5 p$ with CD274 and CCND1 in BRCA (22). The Tumor IMmune Estimation Resource (TIMER) was used to predict the relationship between PD-L1 and CCND1 in BRCA (23).

\section{Cell culture}

MDA-MB-231 cells were purchased from the Cell Bank of FuHeng Biology (China), and HBL-100 cells were obtained as a gift from The Clinical Pharmacy Laboratory of The First Affiliated Hospital of Zhengzhou University. All cells 
Table 1 The primers used for qRT-PCR

\begin{tabular}{lll}
\hline Gene & Forward primer & Reverse primer \\
\hline Actin & GAGCTACGAGCTGCCTGAC & GGTAGTTTCGTGGATGCCACAG \\
$P D-L 1$ & GCTGCACTAATTGTCTATTGGG & CACAGTAATTCGCTTGTAGTCG \\
CCND1 & GCATCTACACCGACAACTCCAT & GTTTGTTCTCCTCCGCCTCT \\
\hline
\end{tabular}

qRT-PCR, quantitative real-time PCR.

were cultured in RPMI-1640 medium (Solarbio; 31800) containing $10 \%$ fetal bovine serum (FBS; BI, 04-001-1A, China), $100 \mathrm{U} / \mathrm{mL}$ penicillin, and $0.1 \mathrm{mg} / \mathrm{mL}$ streptomycin in an incubator with $5 \% \mathrm{CO}_{2}$ at $37^{\circ} \mathrm{C}$.

\section{Clinical BRCA specimens}

Freshly excised BRCA tissues $(\mathrm{N}=33)$ and adjacent noncancerous tissues $(\mathrm{N}=18)$ were collected from The First Affiliated Hospital of Zhengzhou University. All procedures performed in this study involving human participants were in accordance with the Declaration of Helsinki (as revised in 2013). This study was approved by the Ethics Committee of The First Affiliated Hospital of Zhengzhou University (No. 2020-KY-449), and informed consent was obtained from all patients prior to surgery.

\section{Cell transfection}

The mimics/inhibitors of miR-93-5p, siCCND1, siPD-L1, negative control reagent, and plasmids used for the overexpression of PD-L1 were purchased from GenePharma (Shanghai, China). Cells were seeded at a density of $(2.0-3.0) \times 10^{5}$ cells/well in 6 -well plates, and miRNA mimics, inhibitors, siRNAs, and plasmids were subsequently transfected into cells using si-mate or Lipo2000 purchased from GenePharma (Shanghai, China) according to the manufacturer's instructions. Culture medium was changed $24 \mathrm{~h}$ after transfection.

\section{$R N A$ isolation and $c D N A$ conversion}

Total RNA was isolated from cells $24 \mathrm{~h}$ posttransfection using TRIzol (Life Technologies, 248202). The isolated RNA was quantified using a spectrophotometer (NanoDrop 2000, Applied Biosystems), and $1 \mu \mathrm{g}$ RNA was reverse transcribed into cDNA using the PrimeScript ${ }^{\mathrm{TM}} \mathrm{RT}$ reagent Kit with gDNA Eraser (Perfect Real Time) (RR047A, Takara, Kusatsu, Japan) according to the manufacturer's instructions.

\section{Quantitative real-time PCR}

Gene expression levels were quantified by quantitative realtime PCR (qRT-PCR) using TB Green ${ }^{\circledR}$ Premix Ex Taq ${ }^{\text {TM }}$ II (Tli RNaseH Plus) (RR820A, Takara, Kusatsu, Japan) in MicroAmp ${ }^{\mathrm{TM}}$ Fast 8-Tube Strip/MicroAmp ${ }^{\mathrm{TM}}$ Optical 8-Cap Strips (4358293/4323032, Applied Biosystems) on a StepOnePlus ${ }^{\mathrm{TM}}$ real-time PCR system (Applied Biosystems). All reactions were run in triplicate, and the comparative threshold method $\left(2^{-\Delta \Delta \mathrm{C}_{t}}\right)$ was used to calculate results. Expression levels of $m i R-93-5 p$ were normalized to $\mathrm{U} 6$, and the other gene expression levels were normalized to actin. The primers used in this study are summarized in Table 1.

\section{Dual-luciferase reporter assay}

The 3'-UTRs of PD-L1/CCND1 were amplified and inserted into the pSI-Check2 (Hanbio Biotechnology) vector to construct wild-type (wt) vectors. Luciferase activity was measured using the Reporter Assay System Kit (017319, Promega), and "relative luciferase activity" was calculated by comparing the ratio of firefly luciferase activity to Renilla luciferase activity.

\section{Cell proliferation}

Twenty-four hours after transfection, cells were seeded into 96-well plates at a density of $2.5 \times 10^{3}$ cells/well. The cells were cultured in an incubator with $5 \% \mathrm{CO}_{2}$ at $37^{\circ} \mathrm{C}$ for $24 \mathrm{~h}$. Subsequently, $10 \mu \mathrm{L}$ of Cell Counting Kit-8 (CCK-8) solution (Meilunbio ${ }^{\circledR}$, China) was added to each well, followed by incubation for $2 \mathrm{~h}$ in an incubator with $5 \% \mathrm{CO}_{2}$ at $37^{\circ} \mathrm{C}$. The optical density (OD) value at a wavelength of $450 \mathrm{~nm}$ was measured using a SpectraMax ${ }^{\circledR} \mathrm{i} 3 \mathrm{x}$ (Molecular Devices). The cell proliferation rate was calculated as follows: (OD value in the experimental group - OD value in the blank control group)/(OD value in the control group - OD value in the blank control group) $\times 100 \%$. 


\section{Transwell assays}

Cells were harvested 24 h post-transfection. Cells $\left(2 \times 10^{4}\right)$ resuspended in $200 \mu \mathrm{L}$ of serum-free RPMI medium were then seeded into the upper chamber of hydrated polycarbonate membrane insets with $8 \mu \mathrm{m}$ pores (Corning, NY, USA) and transferred to hydrated Matrigel (1:50) invasion chambers (3422, Corning, NY, USA) for the invasion assay (24 h). Subsequently, cells were fixed in 100\% methanol followed by staining with crystal violet $(0.1 \%$ $\mathrm{w} / \mathrm{v})$. To determine the number of cells that had invaded, images from the center and four peripheral fields on the membrane were captured using an inverted microscope at 10x magnification and quantified.

\section{Wound-healing assay}

Cells were seeded onto a 6-well plate and incubated at $37^{\circ} \mathrm{C}$. When cell confluence reached approximately $100 \%$, a $10 \mu \mathrm{L}$ sterilized tip was used to carefully create a line in the cell monolayer. Cells were then imaged in each well and marked differently for subsequent retrieval, and this time point was set as $0 \mathrm{~h}$. After $24 \mathrm{~h}$ of culture at $37^{\circ} \mathrm{C}$, culture medium was removed. Cell debris was removed by rinsing the plate with phosphate buffered saline (PBS) three times, and culture medium containing $2 \%$ fetal bovine serum (FBS) was added. The percentage of scratched zone closure at $24 \mathrm{~h}$ compared to time at $0 \mathrm{~h}$ was measured using Image software. This experiment was performed in triplicate.

\section{Flow cytometry}

Cell cycle and apoptosis analyses were performed using flow cytometry. Briefly, cells were seeded into 6-well plates at $2 \times 10^{5}$ cells/well. Twelve hours later, the cells were treated with specific reagents. Twenty-four hours after transfection, the culture medium in each well was changed. A cell cycle staining kit (KGA511-KGA51, KeyGEN Biotech, China) was used to assess the cell cycle, and an Annexin V-FITC/PI apoptosis kit (556547, BD Biosciences, San Jose, CA, USA) was used to measure apoptosis following the manufacturer's instructions. Flow cytometry analysis was performed in a BD FACSCanto flow cytometry system.

\section{Immunobistochemistry staining}

Paraffin-embedded tissue sections were heated at $60{ }^{\circ} \mathrm{C}$ overnight, treated with xylene and ethanol in gradient concentrations, repaired with EDTA (pH 9.0), incubated with $3 \% \mathrm{H}_{2} \mathrm{O}_{2}$ at room temperature for $10 \mathrm{~min}$, and finally blocked in $3 \%$ bovine serum albumin (BSA) at room temperature for $30 \mathrm{~min}$. The blocked tissue sections were then incubated with anti-PD-L1 antibody (1:200) and antiCCND antibody $(1: 200)$ at $4{ }^{\circ} \mathrm{C}$ overnight. Incubation was terminated washing three times with $\mathrm{PBS}(\mathrm{pH}$ 7.4). Target proteins were probed by incubation with secondary antibodies at room temperature for $50 \mathrm{~min}$. Sections were immersed in DAB, stained with hematoxylin, dehydrated in gradient concentrations of ethanol, mounted in neutral balsam, and observed under a microscope. Slides were images using an optical microscope (Olympus).

\section{Western blot analysis}

Protein was extracted $48 \mathrm{~h}$ posttransfection using RIPA buffer (CWBIO, China) and quantified using a BCA protein assay kit (Solarbio, China). Each sample containing $30 \mu \mathrm{g}$ of protein was denatured at $95{ }^{\circ} \mathrm{C}$ for $10 \mathrm{~min}$ and separated on 4-20\% SDS-PAGE gels (Bio-Rad Laboratories). Proteins were transferred onto PVDF membranes (Millipore, NY, USA), and the membranes were blocked in quick sealing fluid for $10 \mathrm{~min}$ at room temperature, washed with $1 \times$ TBST, and then incubated overnight at $4{ }^{\circ} \mathrm{C}$ with the following anti-human primary antibodies: $\beta$-actin $(1: 1,000$, \#3700, CST), PD-L1 (1:1,000, \#13684, CST), E-cadherin (1:1,000, \#3195, CST), phospho- $\beta$-catenin (1:1,000, \#5651, CST), vimentin (1:1,000, \#5741, CST), CCND1 (1:1,000, ab134175, Abcam), CDK1 (1:50,000, ab133327, Abcam), Cdk4 (1:1,000, ab108357, Abcam), and cyclin B1 (1:50,000, ab32053, Abcam). The next day, membranes were incubated with secondary antibodies at room temperature for $1 \mathrm{~h}$. Finally, the proteins were detected using a LI-COR Odyssey device (Gene Company Limited), and the intensity of the target bands was quantified using ImageJ software. This experiment was repeated three times.

\section{In situ bybridization}

Paraffin-embedded tissue sections were heated at $60{ }^{\circ} \mathrm{C}$ for $1 \mathrm{~h}$, treated with xylene and ethanol in gradient concentrations, digested with Proteinase K, dehydrated in ethanol of gradient concentrations, hybridized with the probe of human U6/miR-93-5p purchased from Bioster (Wuhan, China), and washed in SSC of gradient concentrations. The slides were subsequently treated using a BCIP/NBT substrate kit, mounted in neutral balsam, and observed under a microscope. 


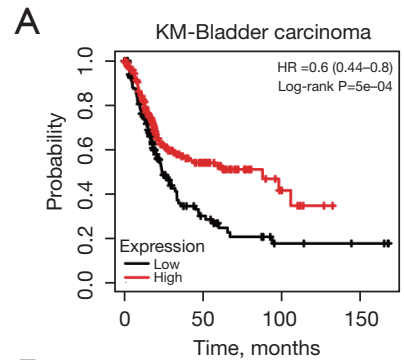

$E$

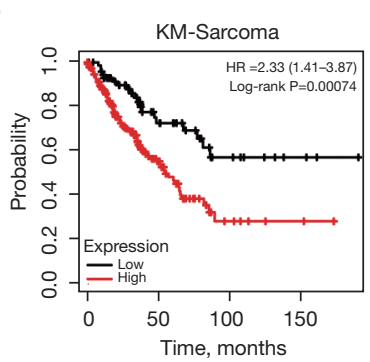

B

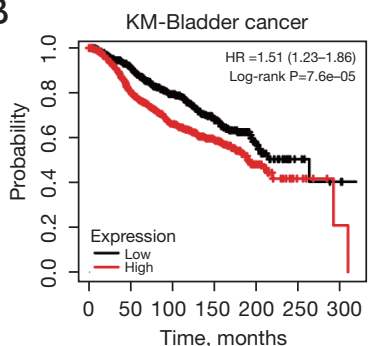

F

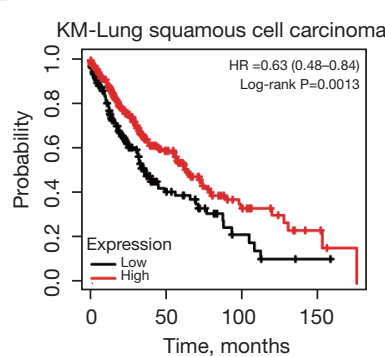

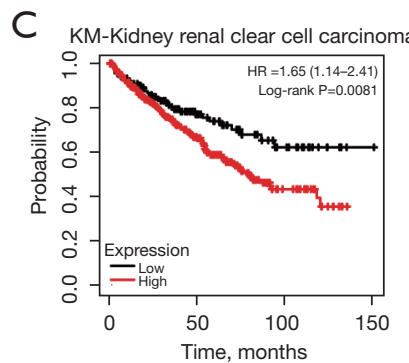

G

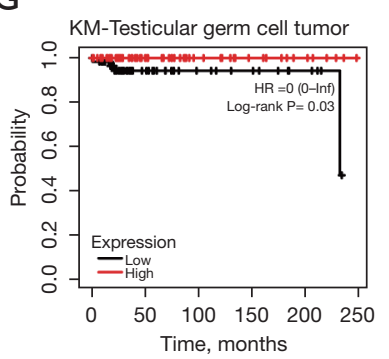

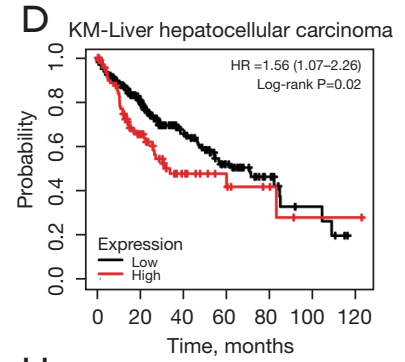

$\mathrm{H}$

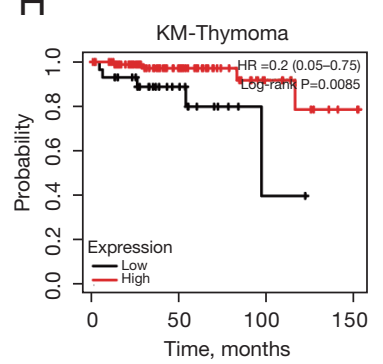

Figure 1 MiR-93-5p produces distinct prognosis outcomes in different tumors. (A-E) High expression of miR-93-5p conveys poor prognosis in bladder carcinoma, breast cancer, kidney renal clear cell carcinoma, liver hepatocellular carcinoma, and sarcoma according to the Kaplan-Meier Plotter datasets; (F-H) high expression of miR-93-5p conveys good prognosis in lung squamous cell carcinoma, testicular germ cell tumor and thymoma according to the Kaplan-Meier Plotter datasets.

\section{Statistical analysis}

Statistical analysis was performed using GraphPad Prism 8.0 software. Experiments were performed in triplicate and repeated at least twice. Two-tailed Student's $t$-test was used to evaluate differences between two groups. Bars indicate the mean \pm standard deviation (SD) of three independent replicates. A P value $<0.05$ was considered statistically significant.

\section{Results}

\section{The expression status of miR-93 is associated with the} prognosis and immunoregulation of tumors

By analyzing the Kaplan-Meier Plotter datasets, we found that the expression data of $m i R-93$ generated an antipodal prognosis in different tumors. For example, high expression of $m i R-93$ was associated with poor prognosis in bladder carcinoma, BRCA, kidney renal clear cell carcinoma, liver hepatocellular carcinoma, and sarcoma (Figure 1A-1E). However, low expression levels of $m i R-93$ were positively correlated with poor prognosis in lung squamous cell carcinoma, testicular germ cell tumor, and thymoma (Figure $1 F-1 H$ ). Therefore, it is important to study the specific mechanisms of action of $m i R-93$. To this end, we predicted the targeted genes of miR-93 using TargetScan 7.2 and miWALK3.0 and obtained 5,453 and 5,078 target genes, respectively. We then intersected the two datasets using Bioinformatics \& Evolutionary Genomics (Figure 2A), and for the target genes that intersected, enrichment analysis was performed using Metascape. From the first 100 enrichment pathways and 20 clusters displayed, we learned that most of the genes were enriched in herpes simplex virus 1 infection and negative regulation of intracellular signal transduction (Figure 2B,2C). However, based on the results of enrichment analysis, we also found that miR-93 regulates the immune response signaling pathway, $\mathrm{T}$ cell receptor and costimulatory and transmembrane receptor protein tyrosine kinase signaling pathways. In addition, we performed cluster analysis for the target genes using DisGeNET, a comprehensive platform integrating information on human diseaseassociated genes and variants, and PaGenBase, a pattern gene database for the global and dynamic understanding of gene function in Metascape, and identified partial target genes clustered in breast adenocarcinoma that were thymus tissue-specific (Figure 2D,2E). Hence, it is thought that miR93 may regulate neoplastic processes and influence tumor immunoregulatory functions.

\section{The prognosis and expression of CCND1/PD-L1 in carcinomas}

CCND1 is a known oncoprotein. We analyzed the 
A

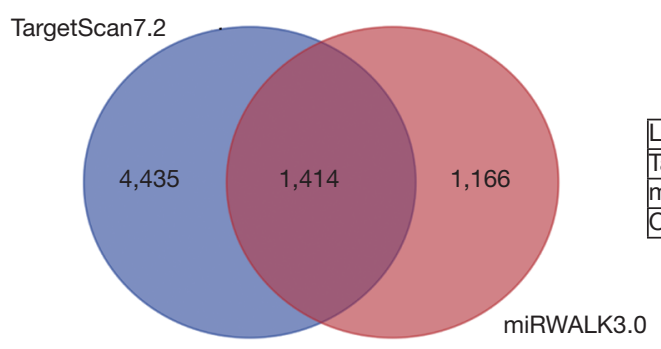

B

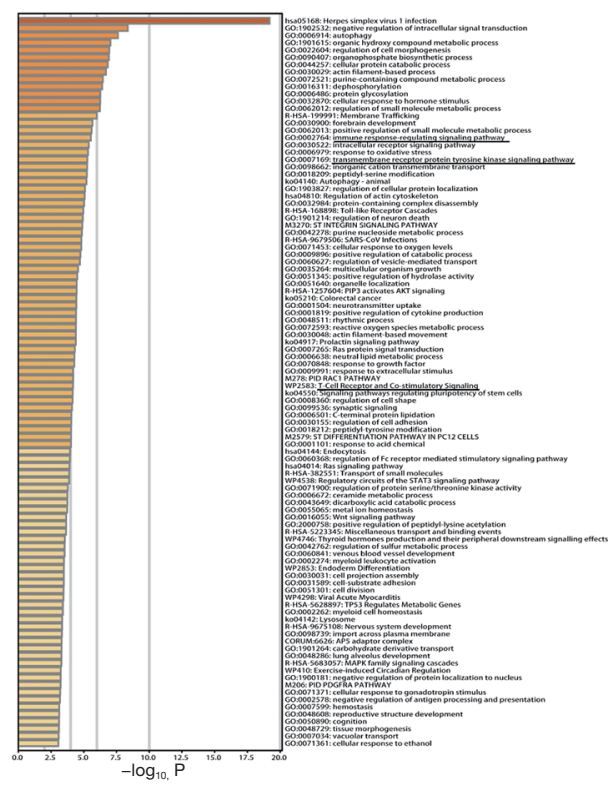

\begin{tabular}{|l|l|l|}
\hline List names & Numbe of elements & Number of unique elements \\
\hline TargetScan & 5,853 & 5,849 \\
\hline miRWALK3.0 & 5,078 & 2,580 \\
\hline Overall number of unique elements & 8,429 \\
\hline
\end{tabular}

C

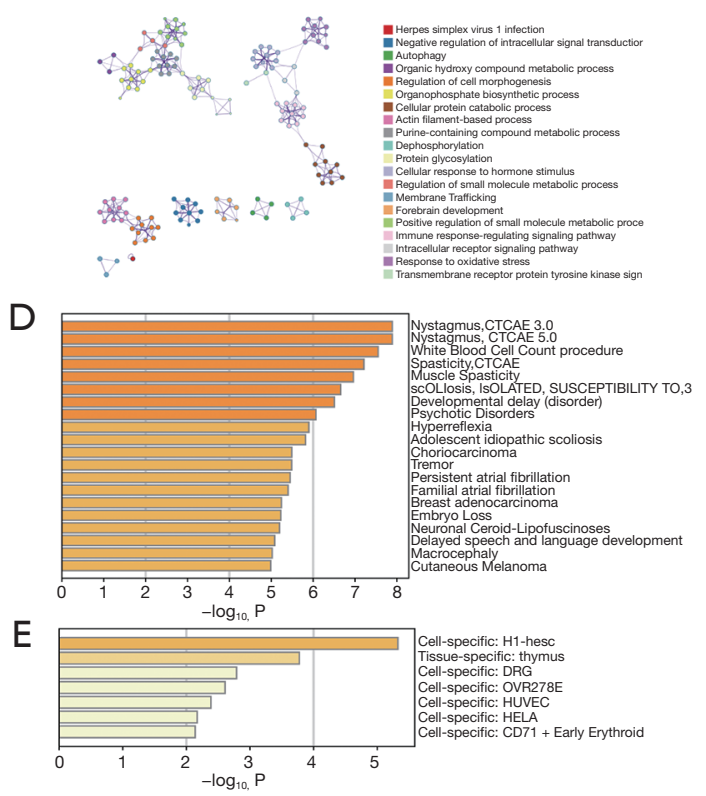

Figure 2 Enrichment analysis of target genes of the miR-93-5p pathway indicate that miR-93-5p regulates the immune response signaling pathway. (A) Venn diagram of 5,453 target genes of hsa-miR-93-5p predicted by TargetScan 7.2 and 5,078 target genes of hsa-miR-935p predicted by miWALK3.0; (B,C) displaying the first 100 enrichment pathways and 20 clusters of enrichment analysis implied that miR93-5p regulates the immune response signaling pathway and the $\mathrm{T}$ cell receptor and costimulatory and transmembrane receptor protein tyrosine kinase signaling pathways; (D,E) cluster analysis of the target genes according to DisGeNET and PaGenBase emphasized target genes clustered in breast adenocarcinoma and tissue-specific thymus.

relationship between CCND1 expression and tumor prognosis using Kaplan-Meier Plotter datasets. As shown in Figure 3, high expression levels of CCND1 were correlated with poor prognosis in BRCA, head-neck squamous cell carcinoma, lung adenocarcinoma, lung squamous cell carcinoma, ovarian cancer, pancreatic ductal adenocarcinoma, and thymoma (Figure $3 A-3 G$ ). However, low expression levels of CCND1 were correlated with poor prognosis in kidney renal clear cell carcinoma, stomach adenocarcinoma, thyroid carcinoma, and uterine corpus endometrial carcinoma (Figure 3H-3K). Afterward, we analyzed expression levels of CCND1 based on sample type and BRCA subtype using UALCAN and found that expression levels of CCND1 were increased in the tumor with respect to sample types (Figure $3 L$ ) compared to the noncancerous breast tissue group, with high expression levels in the luminal subtype and low levels in the HER2 and triple negative BRCA (TNBC) subtypes (Figure 3M). Furthermore, we observed that there was a significant negative correlation between $m i R-93-5 p$ and CCND1 based on the ENCORI datasets (Figure $3 \mathrm{~N}$ ).

PD-L1 is an immunosuppressive protein expressed on tumor cells. By analyzing the Kaplan-Meier Plotter datasets shown in Figure 4, we found that expression levels of PD-L1 

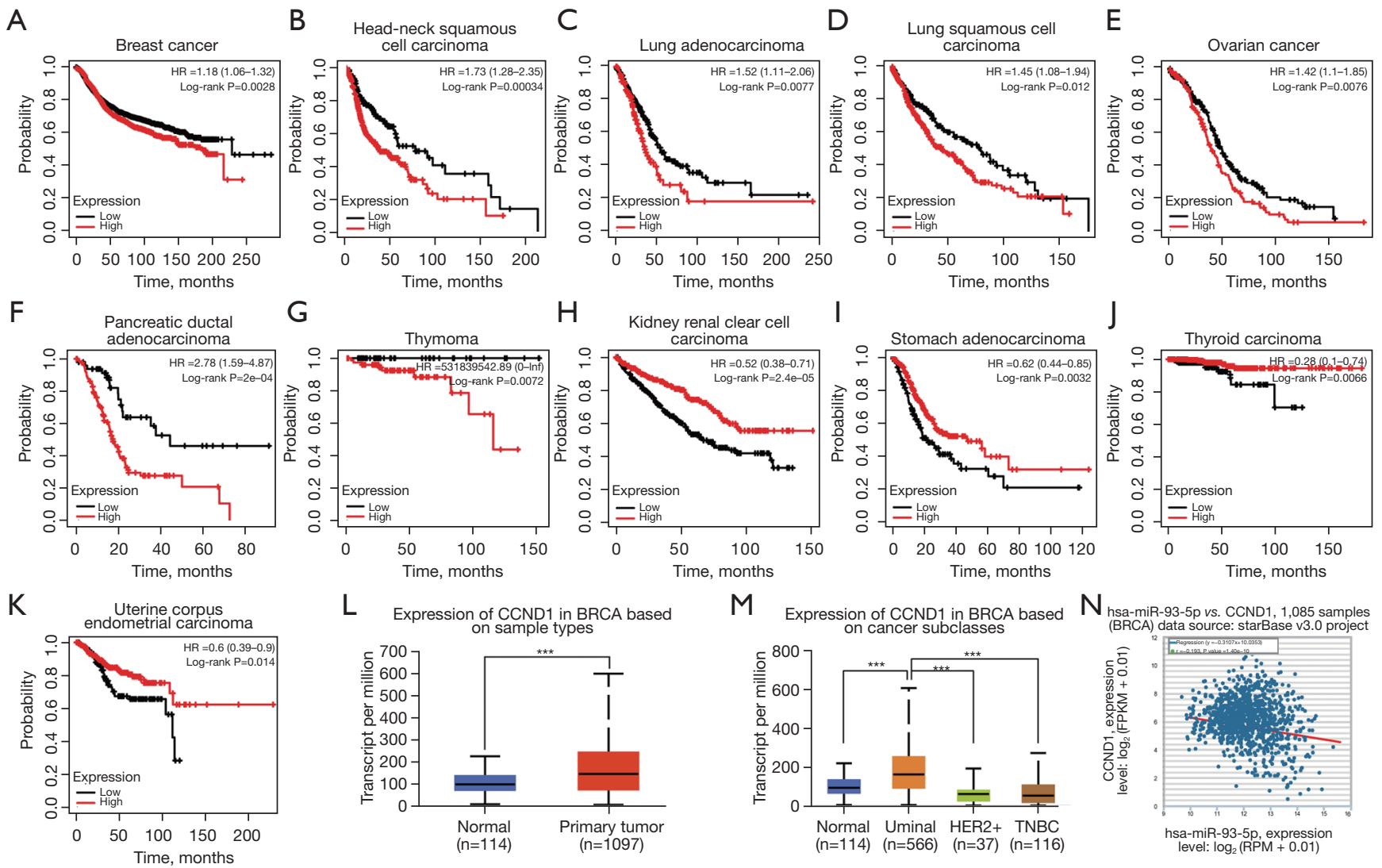

Figure 3 CCND1 generates different prognostic outcomes in various tumors. Expression of CCND1 is differentially increased in BRCA in distinct subclasses of BRCA, and CCND1 was negatively related to miR-93-5p. (A-G) High expression of CCND1 resulted in poor prognosis in pancreatic ductal adenocarcinoma, testicular germ cell tumor, and thymoma according to the Kaplan-Meier Plotter datasets; (H-K) high expression of CCND1 conveyed good prognosis in kidney renal clear cell carcinoma, stomach adenocarcinoma, thyroid carcinoma, and uterine corpus endometrial carcinoma according to Kaplan-Meier Plotter datasets; (L) CCND1 was increased in BRCA according to UALCAN datasets; (M) CCND1 was increased in the luminal subtype and decreased in the HER2 ${ }^{+}$and TNBA subtypes of BRCA according to UALCAN datasets; (N) CCND1 was negatively correlated with miR-93-5p. ${ }^{* * *} \mathrm{P}<0.001$. BRCA, breast cancer.

exhibited different prognoses, with high expression levels of PD-L1 correlating with poor prognosis in pancreatic ductal adenocarcinoma, testicular germ cell tumor, and thymoma (Figure 4A-4C) and low expression levels of PD-L1 correlating with poor prognosis in BRCA, kidney renal clear cell carcinoma, liver hepatocellular carcinoma, ovarian cancer, sarcoma, and uterine corpus endometrial carcinoma (Figure 4D-4I). Next, we analyzed expression levels of PD-L1 based on sample type and BRCA subtype using UALCAN and found that expression levels of PD-L1 were reduced in the tumor in terms of sample types (Figure 47) while expression levels of PD-L1 were high in HER2 ${ }^{+}$and low in luminal TNBC subtypes (Figure $4 K$ ) compared to the noncancerous breast tissue group. Moreover, we observed a negative correlation between $m i R-93-5 p$ and PD-L1 using
ENCORI, even though the difference was not statistically significant (Figure 4L).

\section{MiR-93-5p targets PD-L1 and suppresses CCND1}

To investigate the relationships of $m i R-93-5 p$ with PD-L1 and CCND1, we first assessed mRNA and protein expression levels of $m i R-93-5 p$, PD-L1, and CCND1. qRT-PCR and western blot analysis revealed decreased expression levels of $m i R-93-5 p$ and increased expression levels of PD-L1 and CCND1 in MDA-MB-231 cells (Figure $5 A-5 D$ ). We subsequently overexpressed $m i R-93-5 p$ in MDA-MB-231 cells by transfecting $m i R-93-5 p$ mimics and found that expression of PD-L1 and CCND1 was attenuated (Figure $5 E-5 H$ ). Then, we 


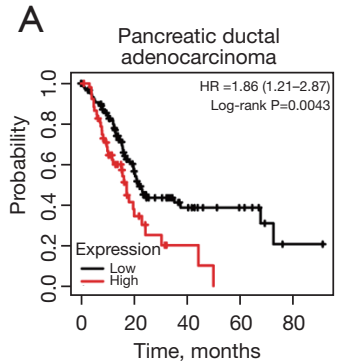

F Liver hepatocellular
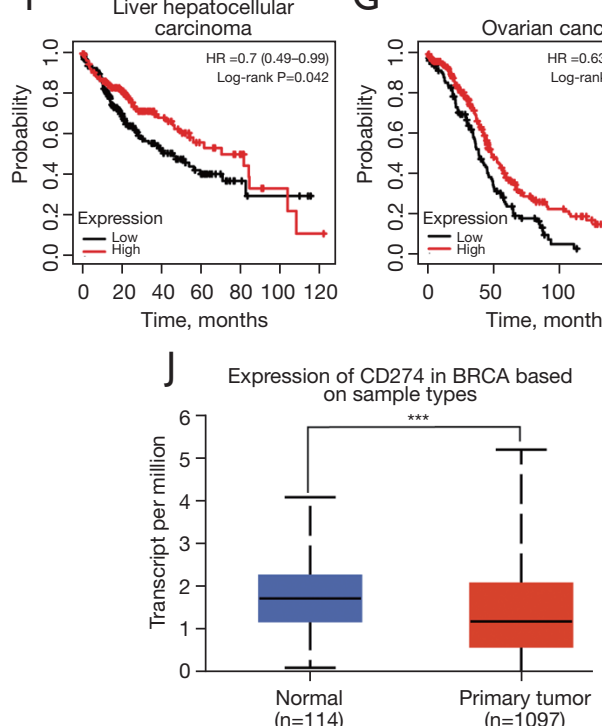

G
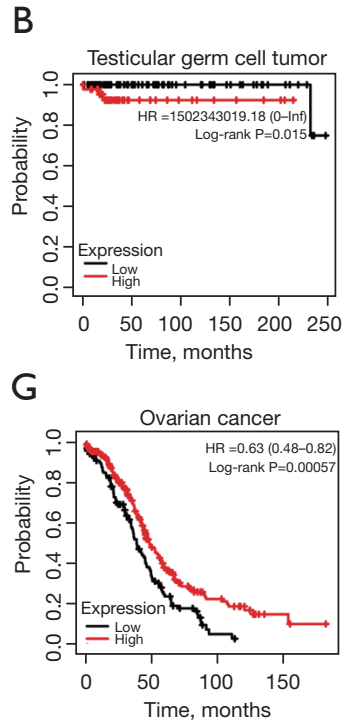

K

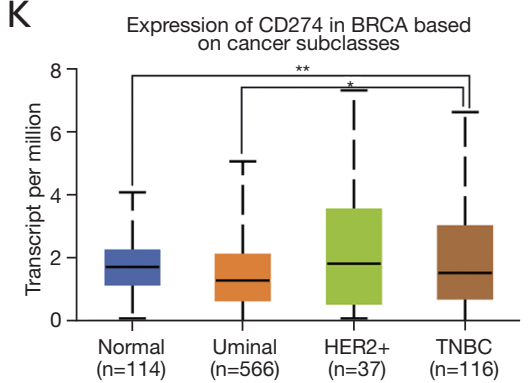

C
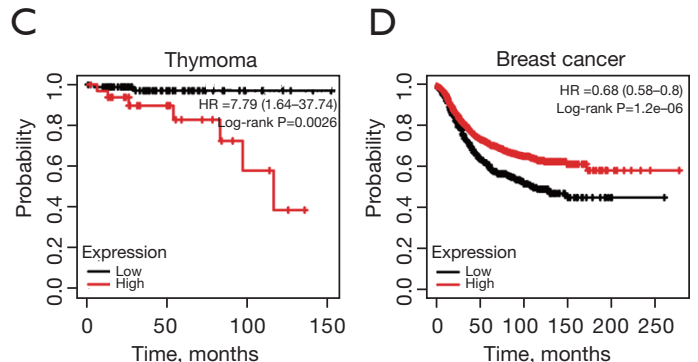

$\mathrm{H}$

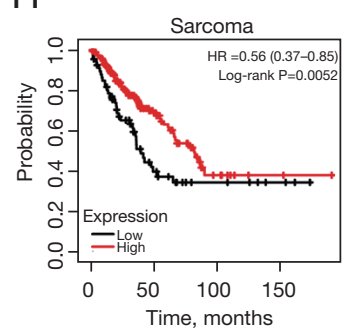

I Uterine corpus endometrial

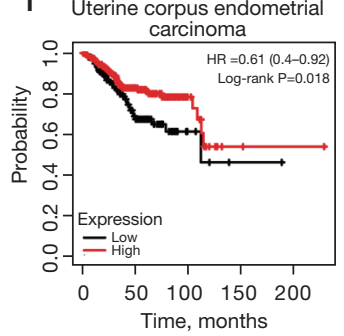

E Kidney renal clear cell

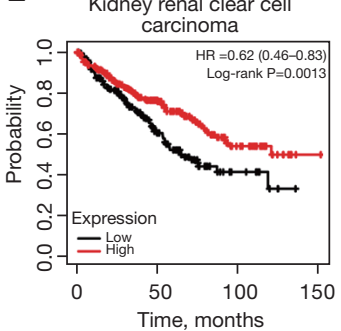

L

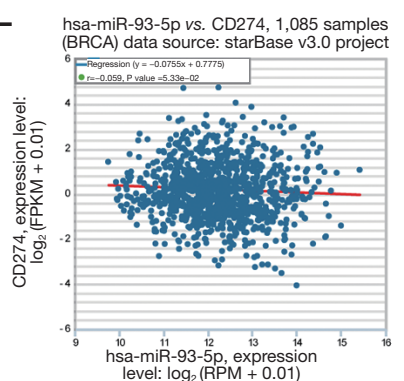

Figure 4 PD-L1 generates differential prognostic outcomes in various tumors, and expression of PD-L is differentially decreased in BRCA in distinct subtypes of BRCA. (A-C) High expression of PD-L1 resulted in poor prognosis in breast cancer, head-neck squamous cell carcinoma, lung adenocarcinoma, lung squamous cell carcinoma, ovarian cancer, pancreatic ductal adenocarcinoma, and thymoma according to Kaplan-Meier Plotter datasets. (D-I) High expression of PD-L1 conveyed good prognosis in breast cancer, kidney renal clear cell carcinoma, liver hepatocellular carcinoma, ovarian cancer, sarcoma, and uterine corpus endometrial carcinoma according to Kaplan-Meier Plotter datasets. (J) PD-L1 was decreased in BRCA according to UALCAN datasets. (K) PD-L1 was decreased in luminal and triple negative breast cancer (TNBC) subtypes of BRCA according to UALCAN datasets ( $\mathrm{P}<0.01$ ). (L) PD-L1 was negatively correlated with miR-93-5p, but the difference was not statistically significant. ${ }^{* *} \mathrm{P}<0.01,{ }^{* * *} \mathrm{P}<0.001$. BRCA, breast cancer.

inhibited the expression of $m i R-93-5 p$ in MDA-MB-231 cells by transfecting $m i R-93-5 p$ inhibitors, and qRT-PCR and western blot indicated that expression of PD-L1 and CCND1 was augmented (Figure $5 I-5 L$ ). To determine whether miR-93-5p directly binds to the 3'-UTRs of $\mathrm{PD}-\mathrm{L} 1 / \mathrm{CCND} 1$, we conducted a dual-luciferase reporter assay, and the results showed that $m i R-93-5 p$ decreased the luciferase activities of PD-L1 but not those of CCND1 compared to wt, implying that miR-93-5p directly targets PD-L1 (Figure 5M-5P).

PD-L1 is localized in the cytoplasm and membrane, and CCND1 is localized in the cytoplasm and nucleus based on the immunofluorescence results of PD-L1 and CCND1 from
The Human Protein Atlas (HPA) (Figure 6A). To examine the dynamics of PD-L1 and CCND1, cytoplasm, cytomembrane, and nuclear proteins from BRCA cells transfected with $m i R-93-5 p$ mimics/inhibitors were separately extracted, and protein expression levels of PD-L1 and CCND1 were assessed by western blot. Our results showed that PD-L1 was primarily localized in the membrane and that CCND1 was primarily located in the cytoplasm and nucleus. In response to increasing the concentration of miR-93-5p in MDAMB-231 cells, the concentrations of PD-L1 in the membrane and CCND1 in the cytoplasm were subsequently decreased, and no obvious change was observed in the nucleus. In contrast, MDA-MB-231 cells were transfected with miR-93- 
A

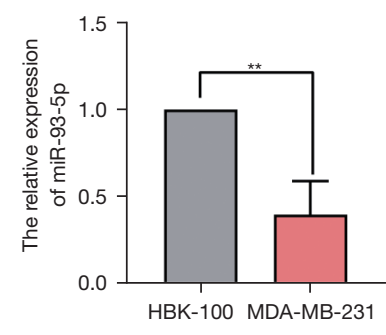

E

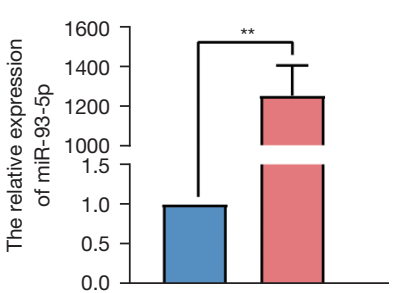

I

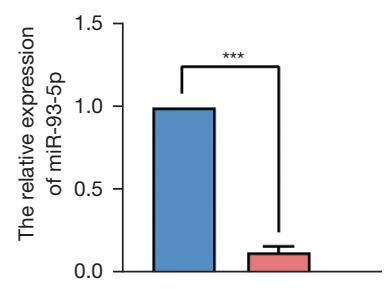

M
B

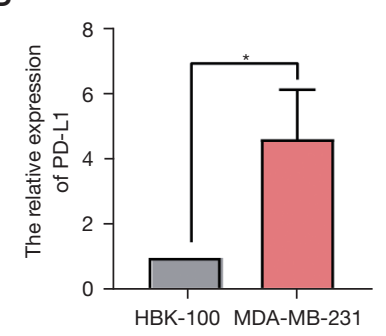

$\mathrm{F}$

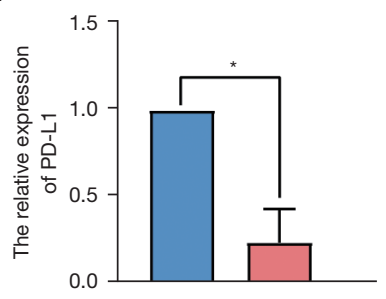

C

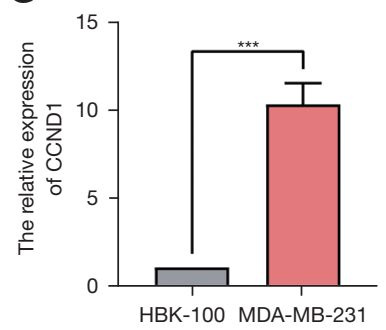

G

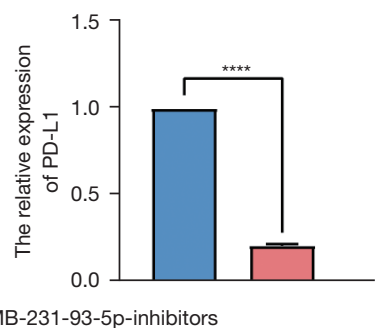

$\mathrm{K}$

J

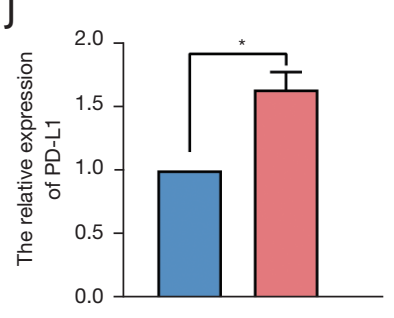

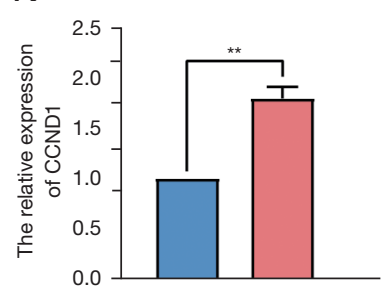

D

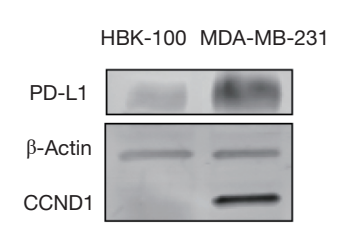

$\mathrm{H}$

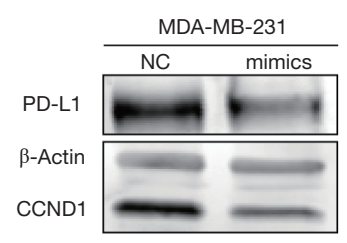

$\mathrm{L}$

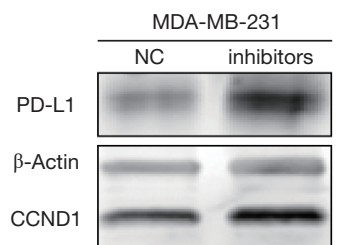

$\square$ MDA-MB-231-NC $\square$ MDA-MB-231-93-5p-inhibitors

0

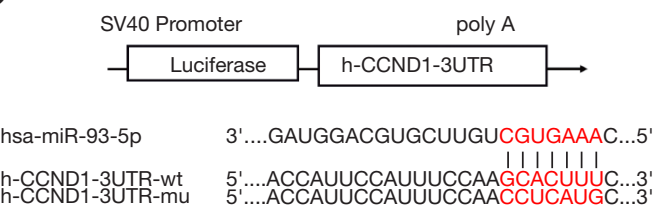

$\mathrm{N}$

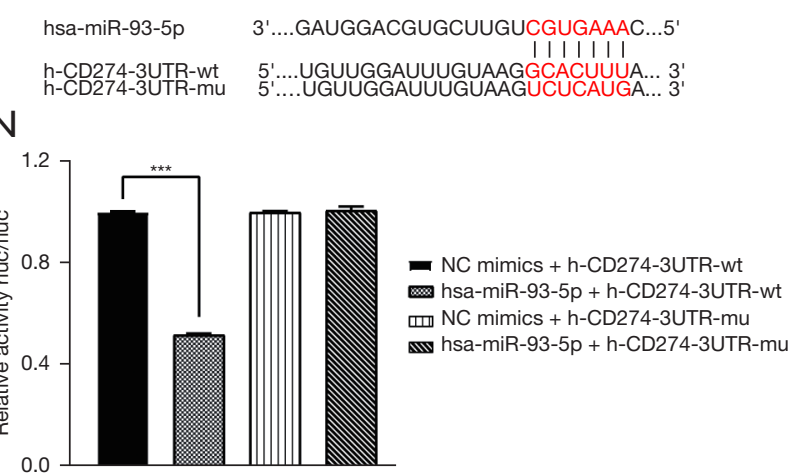

P

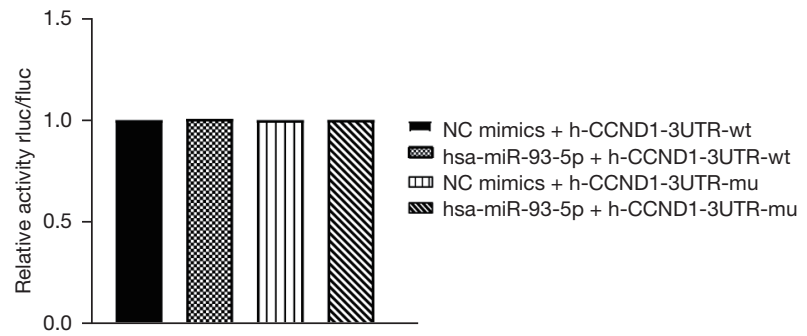

Figure 5 Expression of miR-93-5p is different between MDA-MB-231 and HBL-100 cells and directly targets PD-L1 and CCND1. (A-C) Gene expression levels of miR-93-5p, PD-L1 and CCND1 in MDA-MB-231 and HBL-100 cells; (D) protein expression levels of PD-L1 and CCND1 in MDA-MB-231 and HBL-100 cells; (E-G) gene expression levels of miR-93-5p, PD-L1 and CCND1 in MDA-MB-231 cells transfected with miR-93-5p mimics; $(\mathrm{H})$ protein expression levels of PD-L1 and CCND1 in MDA-MB-231 cells transfected with miR-935p mimics; (I-K) gene expression levels of miR-93-5p, PD-L1 and CCND1 in MDA-MB-231 cells transfected with miR-93-5p inhibitors; (L) protein expression levels of PD-L1 and CCND1 in MDA-MB-231 cells transfected with miR-93-5p inhibitors; (M,N) the luciferase reporter results of miR-93-5p-targeted PD-L1 ( $\mathrm{P}<0.001)$; (O,P) the luciferase reporter results of miR-93-5p-targeted CCND1. * $\mathrm{P}<0.05$, ${ }^{* *} \mathrm{P}<0.01,{ }^{* * *} \mathrm{P}<0.001,{ }^{* * *} \mathrm{P}<0.0001$. 

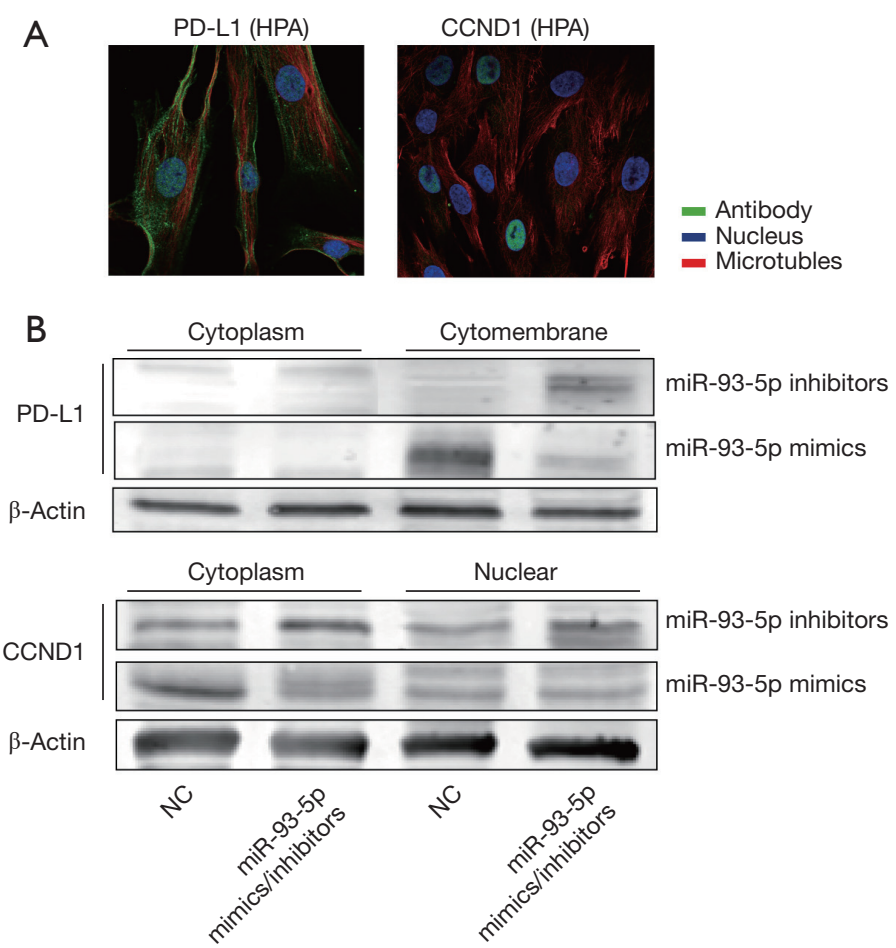

Figure 6 MiR-93-5p regulates PD-L1 in the cytomembrane and regulates CCND1 in the cytoplasm. (A) Expression sites of PD-L1 and CCND1 according to The Human Protein Atlas (HPA); (B) Western blot analysis of the protein expression levels of PD-L1 and CCND1 in MDA-MB-231 cells transfected with miR-93-5p mimics or inhibitors.

$5 p$ inhibitors and exhibited the opposite results (Figure 6B).

\section{MiR-93-5p inhibits proliferation and regulates the migration and invasion of BRCA cells by targeting $P D$ - L1/CCND1}

Based on the previous CCK- 8 results, we found that the growth rate of MDA-MB-231 cells transfected with $m i R$ 93-5p mimics was reduced, while the growth rate of MDAMB-231 cells transfected with miR-93-5p inhibitors was increased (Figure $7 A, 7 B$ ). Next, we examined miR-93$5 p$ 's effects on the cell cycle by flow cytometry and found that MDA-MB-231 cells transfected with $m i R-93-5 p$ mimics were arrested in G0/G1, and the number of cells in $S$ phase was reduced, while the number of G2/M phase MDA-MB-231 cells transfected with miR-93-5p inhibitors was increased (Figure $7 C-7 F$ ). Furthermore, wound healing experiments indicated that miR-93-5p inhibits the migration of MDA-MB-231 cells transfected with mimics and accelerates the migration of MDA-MB-231 cells transfected with inhibitors. Transwell experiments also indicated that $m i R-93-5 p$ inhibits the invasiveness of
MDA-MB-231 cells (Figure 8A-8C). Western blot analysis revealed that expression of E-cadherin and phospho- $\beta$ catenin was enhanced, while that of vimentin was reduced in MDA-MB-231 cells transfected with $m i R-93-5 p$ mimics. The opposite result was observed in MDA-MB-231 cells transfected with miR-93-5p inhibitors (Figure 8D).

\section{PD-L1 and CCND1 are overexpressed and exhibit mutual regulatory effects in BRCA tissues}

We examined expression levels of PD-L1 and CCND1 by IHC in noncancerous breast tissue $(\mathrm{n}=18)$ and BRCA tissue ( $n=33$ ) collected from the First Affiliated Hospital of Zhengzhou University. Compared to the noncancerous breast group, higher expression levels of PD-L1 and CCND1 were observed in the BRCA group (Figure 9A-9C). Moreover, the bioinformatics analysis of TIMER datasets revealed that expression levels of CCND1 were positively correlated with that of PD-L1, in accordance with previous immunohistochemical results. The results from knockdown PD-L1 and CCND1 in MDA-MB-231 cells revealed that expression levels of CCND1 were reduced in MDA- 
A

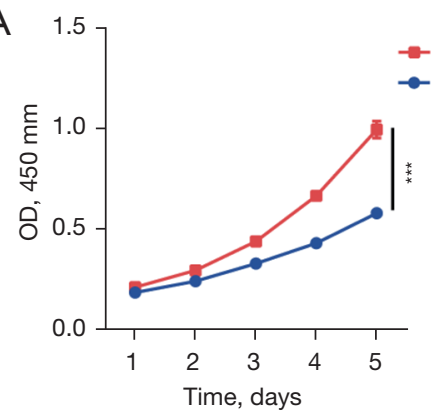

C

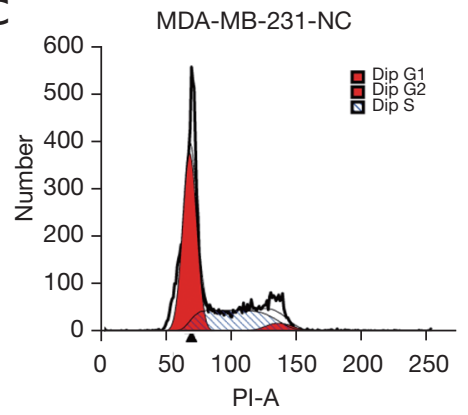

E

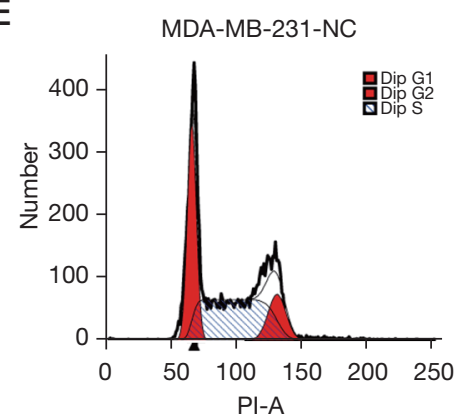

B

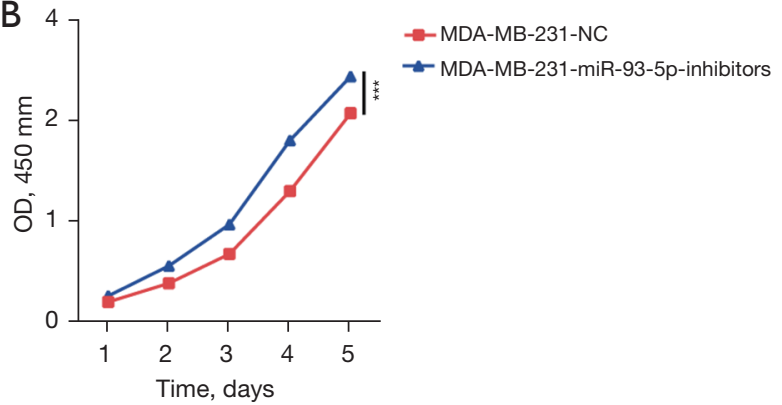

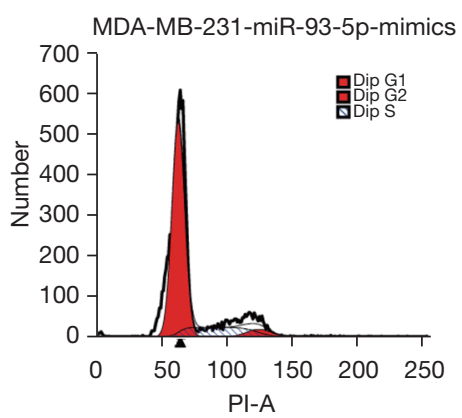

D
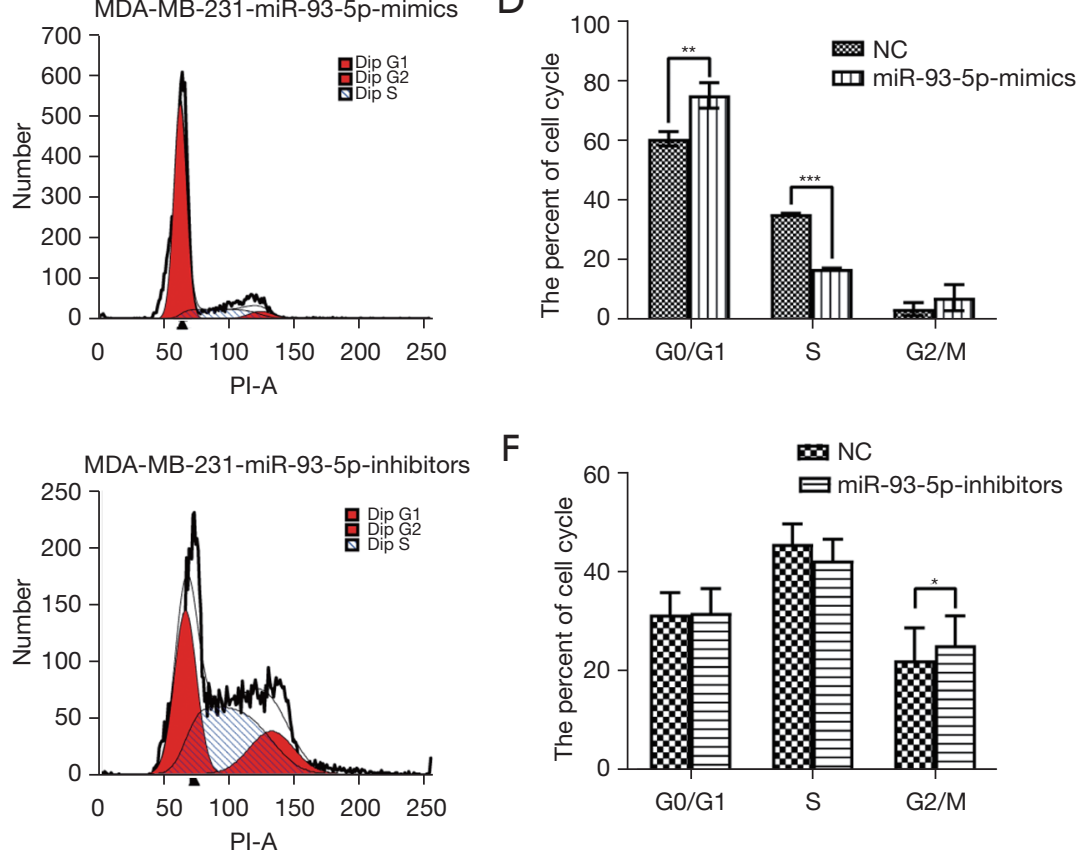

$\mathrm{F}$
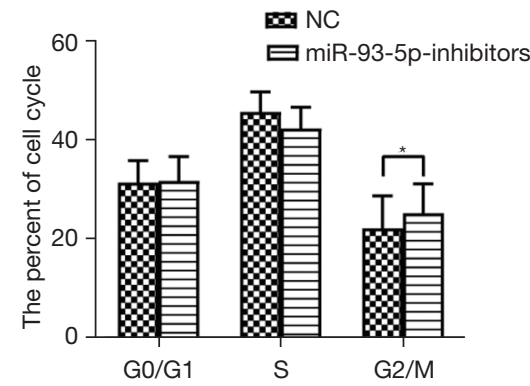

Figure 7 MiR-93-5p suppresses cell proliferation and cell cycle progression in breast cancer cells. (A) The cell growth curves are depicted for MDA-MB-231 cells transfected with miR-93-5p mimics; (B) the cell growth curves are depicted for MDA-MB-231 cells transfected with miR-93-5p inhibitors; (C,D) change in the cell cycle in MDA-MB-231 cells transfected with miR-93-5p mimics; (E,F) changes in the cell cycle in MDA-MB-231 cells transfected with miR-93-5p inhibitors. ${ }^{*} \mathrm{P}<0.05$, ${ }^{* *} \mathrm{P}<0.01,{ }^{* * *} \mathrm{P}<0.001$.

MB-231 cells transfected with si-PD-L1. In contrast, expression levels of PD-L1 were higher in MDA-MB-231 cells transfected with si-CCND1. Likewise, we observed that there were some regions in the BRCA tissue slices in which expression of CCND1 was not detected while that of PD-L1 was observed (Figure 10A-10C). In summary, these results indicate that PD-L1 and CCND1 regulate each other and that there might be a feedback mechanism between these two proteins.

\section{MiR-93-5p regulates tumor immunology by targeting PD-L1}

Previous works have confirmed that $m i R-93-5 p$ regulates immune responses and tumorigenesis by inhibiting the PD-L1/CCND1 signaling pathway. To further explore the relationship of $m i R-93-5 p$ and tumor immunity, we assessed the expression of $m i R-93-5 p$ using ISH. In tissues with low expression of $m i R-93-5 p$, expression of PD-L1 was high, but expression levels of PD-L1 were reduced in tissues with high expression levels of miR-93-5p. At the same time, we found that expression levels of CD8 were the same as that of PD-L1 (Figure 11). Furthermore, according to the TIMER datasets, expression levels of PD-L1 and CD8 were positively correlated in BRCA, consistent with earlier tissue assessments (Figure 12). Finally, we separately analyzed the correlations between clinicopathologic features, including 
A
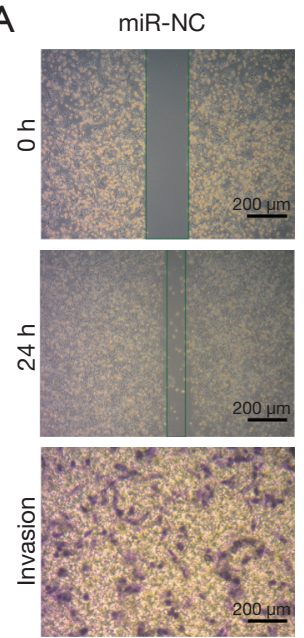

B

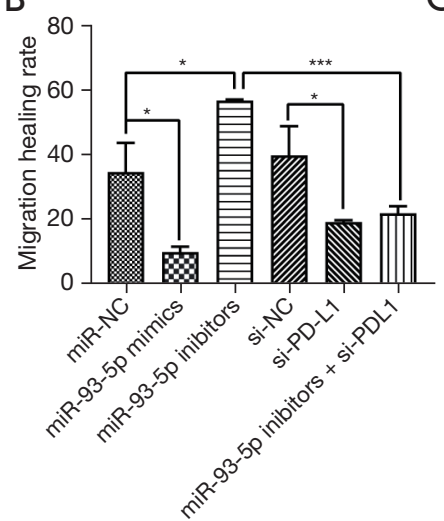

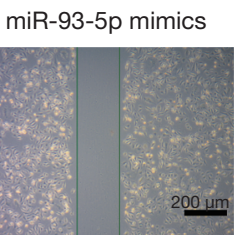
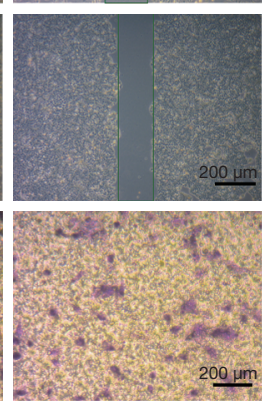

C

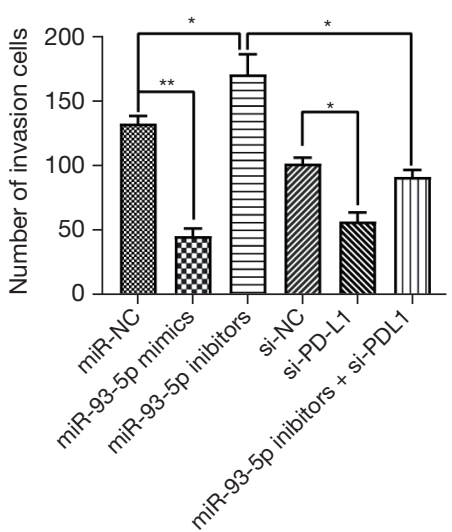

si-NC
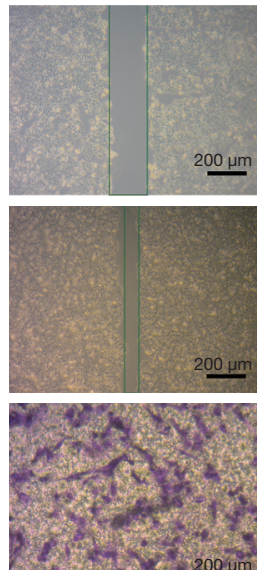
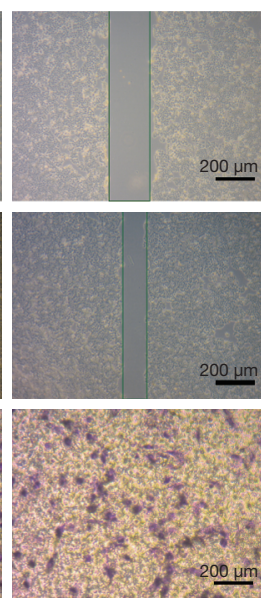

Si-PD-L1
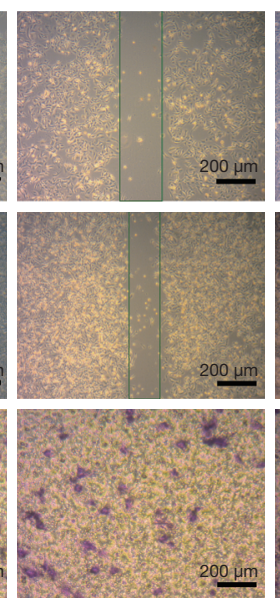

D

miR-93-5p inibitors + si-PDL1
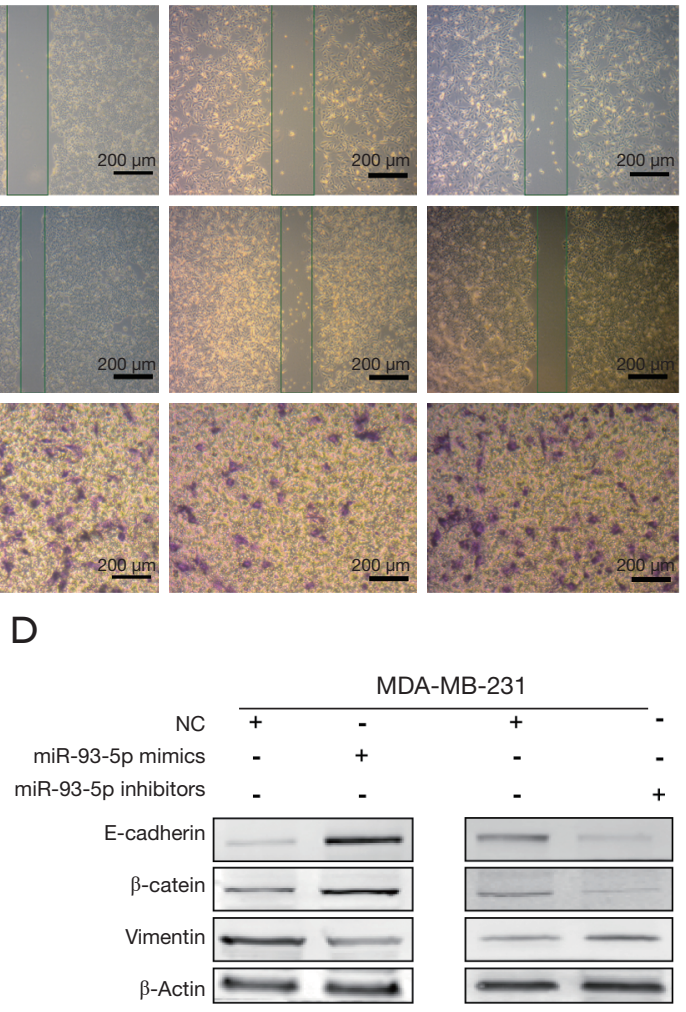

Figure 8 MiR-93-5p inhibits migration and invasion by targeting PD-L1 in breast cancer cells. (A) The cell migration and invasion ability observed by the wound-healing assay and Transwell invasion assay (magnification was $200 \mu \mathrm{m}$ ) and crystal violet staining; (B,C) Statistical analysis of migration and invasion ability; (D) Western blot analysis of migration- and invasion-related proteins in MDA-MB-231 cells transfected with miR-93-5p mimics or miR-93-5p inhibitors. ${ }^{*} \mathrm{P}<0.05,{ }^{* *} \mathrm{P}<0.01,{ }^{* * *} \mathrm{P}<0.001$. PD-L1, programmed cell death-ligand 1.

age, lymph metastasis, pathological grade, T stage, TNM stage, relapse, ER status, PR status, HER2 status and the expression status of $m i R-93-5 p$. The results suggested that expression levels of $m i R-93-5 p$ were not correlated with age, lymph metastasis, pathological grade, $\mathrm{T}$ stage, relapse, ER status, PR status, or HER2 status but was correlated with TNM stage. The correlation between the expression level of $m i R-93-5 p$ and patient survival was not statistically significant in BRCA, likely due to the small sample size (Table 2).

\section{Discussion}

Breast cancer (BRCA) is a threat to women's health worldwide. The two major therapeutic methods for BRCA are locoregional treatment and systemic therapy. However, the histological and molecular characteristics of BRCA can largely influence treatment strategy. Therefore, generating evidence on individuals to fulfil risk-stratified BRCA prevention and screening is urgently needed.

miRNAs are important for various cellular homeostatic functions, and their roles extend to a large number of disease manifestations beyond cancer, such as cardiovascular disease, atherosclerosis, diabetes, and so on. Aberrant expression profiles of miRNAs have been used to identify and classify disease states of cancer or cancer subtypes. For example, miRNAs identified in the early stage of BRCA could be used for discrimination of ER, PR or HER2 status in cancer (24-26). Some tumor suppressive small interfering miRNAs and oncogenic antisense oligonucleotides have 
A A CCND1
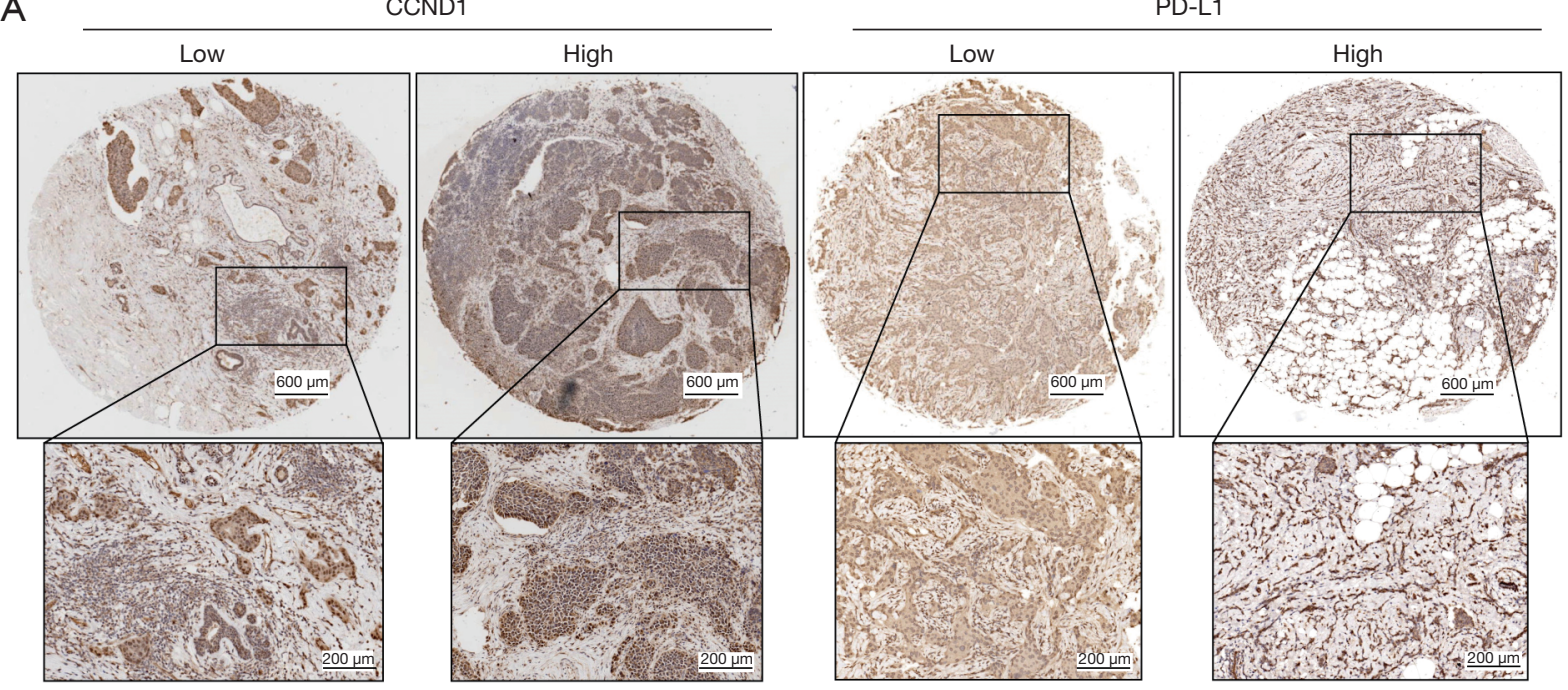

PD-L1

B

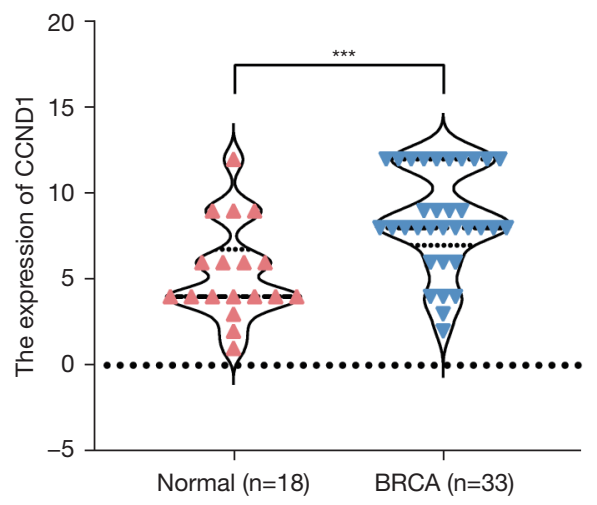

C

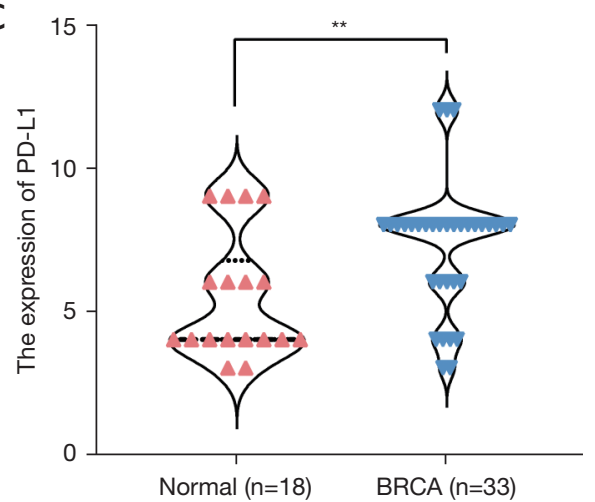

Figure 9 CCND1 and PD-L1 exhibit high expression in breast cancer. (A) Expression of PD-L1 and CCND1 in BRCA (magnification was $200 \mu \mathrm{m}$ and $400 \mu \mathrm{m}$ ) and DAB staining; (B) CCND1 was highly expressed in BRCA tissues compared to noncancerous breast tissues; (C) PD-L1 was highly expressed in BRCA tissues compared to noncancerous breast tissues. ${ }^{* *} \mathrm{P}<0.01,{ }^{* * *} \mathrm{P}<0.001$. PD-L1, programmed cell death-ligand 1; BRCA, breast cancer.

been tested for their anticancer effectiveness, and a few compounds have entered phase I or II clinical trials. However, only a minority (16\%) of those agents can reach phase III trials with far less (10.4\%) finally approved by the $\operatorname{FDA}(26,27)$.

According to the current literature regarding $m i R-93-5 p$, its expression levels and functions vary in different types of tumors. For example, miR-93-5p was expressed at high levels in gastric cancer and devitalized the Hippo signaling pathway to regulate gastric cancer cell progression (28). Likewise, another study claimed that $m i R-93-5 p$ expression was increased in gastric cancer tissues compared to adjacent noncancerous tissues and promoted gastric cancer metastasis by activating the STAT3 signaling pathway (29).
Moreover, another study indicated that $m i R-93-5 p$ was highly expressed in cervical cancer tissues and cells and facilitated cervical cancer progression by targeting the THBS2/MMPS signaling pathway (30). In endometrial carcinoma tissues, miR-93 was overexpressed compared to noncancerous endometrial tissues and boosted epithelialmesenchymal transition (EMT) of endometrial carcinoma cells (31). However, $m i R-93-5 p$ may play the opposite role in other tumors. For instance, glioma studies showed that $m i R-93-5 p$ was downregulated in glioma and inhibited the proliferation and metastasis of glioma cells by targeting MMP2. Colorectal cancer (CRC) studies have suggested that $m i R-93-5 p$ is significantly downregulated in CRC tissues compared to noncancerous tissues and 
A

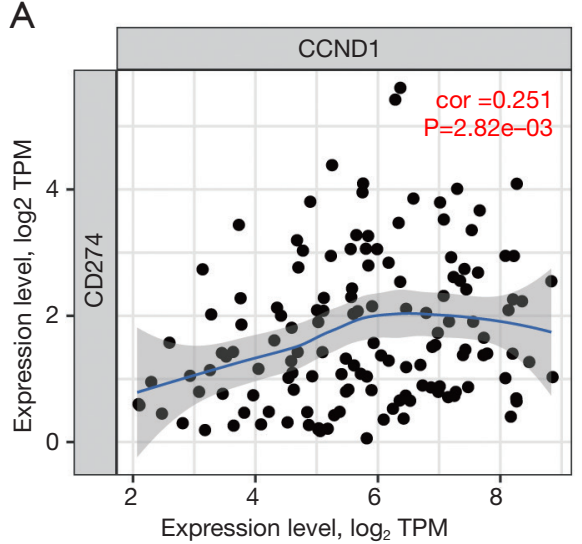

B

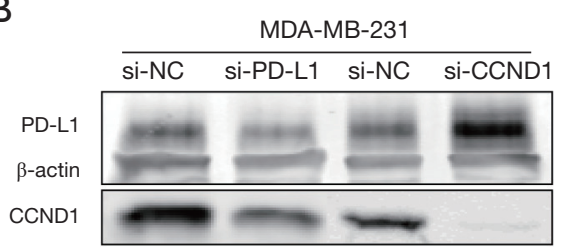

C
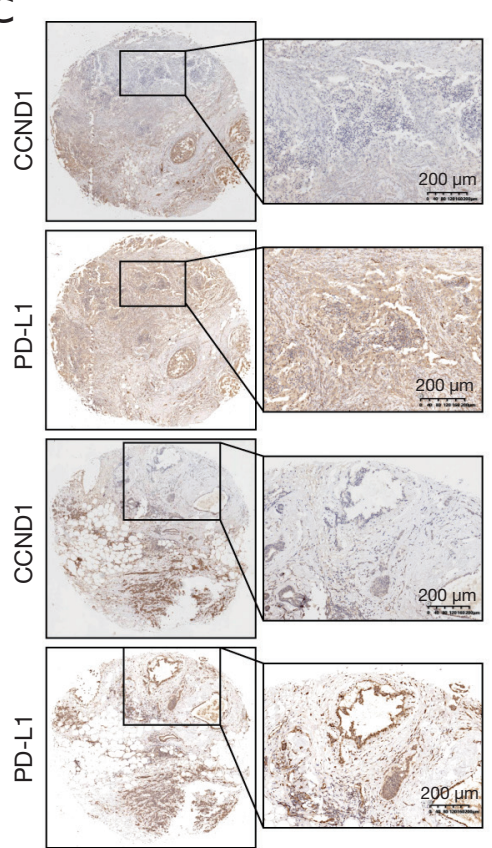

Figure 10 PD-L1 and CCND1 are positively correlated and can regulate each other. (A) CCND1 was positively correlated with PDL1 according to the TIMER datasets; (B) Western blot results indicated that CCND1 was lower in MDA-MB-231 cells transfected with si-PD-L1 and that PD-L1 was higher in MDA-MB-231 cells transfected with si-CCND1; (C) IHC results suggested that expression of CCND1 was absent and that PD-L1 was expressed in some regions of breast cancer tissue sections (magnification was $200 \mu \mathrm{m}$ and $400 \mu \mathrm{m}$ ) and DAB staining. PD-L1, programmed cell death-ligand 1.

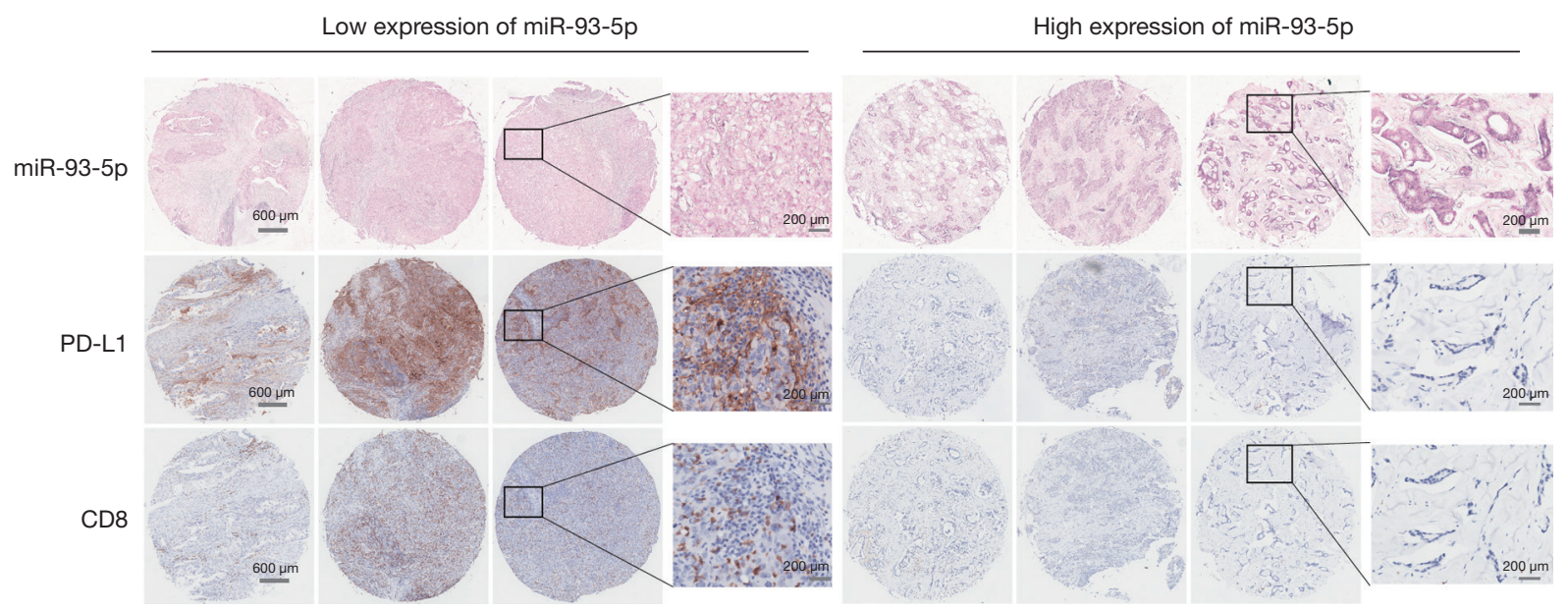

Figure 11 MiR-93-5p regulates tumor immunology by targeting PD-L1. In tissues with low expression of miR-93-5p (BCIP/NBT staining), PD-L1 and CD8 were highly expressed (DAB staining), and in tissues with high expression of miR-93-5p, PD-L1 and CD8 was low. These expression trends were consistent in breast cancer (magnification was 200 and $400 \mu \mathrm{m}$ ). PD-L1, programmed cell death-ligand 1. 


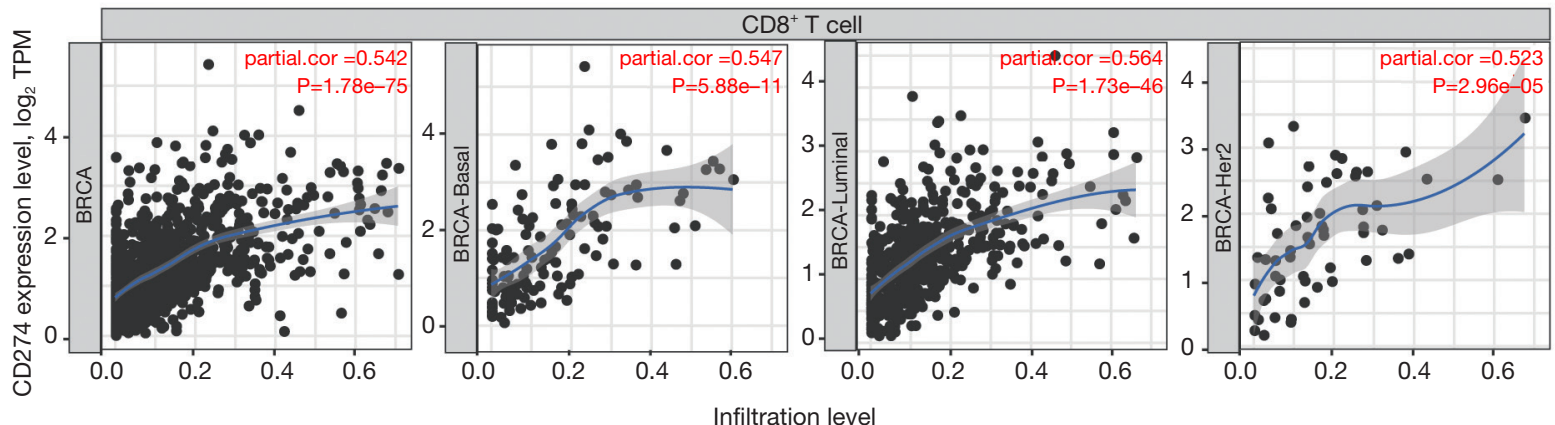

Figure 12 PD-L1 and CD8 are positively correlated in breast cancer according to the TIMER datasets. PD-L1 exhibited a positive correlation with CD8 in basal, luminal and HER2 breast cancer subtypes according to TIMER datasets. PD-L1, programmed cell deathligand 1; BRCA, breast cancer.

exert a tumor suppressor function by targeting PD-L1 in colorectal cancer $(32,33)$. In particular, several studies have reported that miR-93 suppresses tumorigenesis and enhances chemosensitivity by dual targeting of E2F1 and CCND1, inhibiting the invasive potential of triplenegative BRCA cells in vitro via the protein kinase WNK1 in BRCA, and $m i R-93-5 p$ inhibits the EMT of BRCA cells by targeting MKL-1 and STAT3. This indicates that $m i R-93-5 p$ may control signal network nodes to exert its roles $(4,34,35)$. In this study, we found that expression levels of $m i R-93-5 p$ were different in distinct types of BRCA cells. Compared to HBL-100 noncancerous breast cells, MDA-MB-231 BRCA cells, which are a triple-negative BRCA subtype, exhibited downregulated expression levels of $m i R-93-5 p$. In addition, the results suggested that $m i R-93-5 p$ acts on BRCA cell progression by inhibiting BRCA cell proliferation and the cell cycle. In this study, we firstly analyzed the role of $m i R-93-5 p$ in tumor immune regulation and inhibition of migration and invasion through the PD-L1/CCND1 signaling pathway in BRCA. In addition, this study is the first time to elucidate the spatial structure of Mir-93-5p regulating the expression changes of PD-L1/CCND1. $\mathrm{PD}-\mathrm{L} 1$ is a membrane expressed protein, and CCND1 is a cytoplasmic and nuclear expressed protein regulated by $m i R-93-5 p$ in the nucleus. There is also a mutual regulation between PD-L1 and CCND1. PD-L1 is a target of immune examination. In recent years, immunosuppressive therapy has been transformed from non-solid tumor to solid tumor in tumor treatment, and has achieved good therapeutic effect. However, PD-L1 is not a first-line treatment for BRCA in China, and is mainly applied to solid tumors such as non-small cell lung cancer and liver cancer. CCND1 is a known oncogenic protein in various tumors. Therefore, if the immunosuppression and upstream and downstream regulation of CCND1 can be clearly studied, it will provide direction and strategy for its clinical application and individualized treatment of patients.

In this study, we investigated the relationship among CCND1, miR-93-5p, PD-L1 and EMT. Tobin et al. claimed that CCND1 promotes the migration of BRCA cells and that this promotion was abolished by Id1 siRNA treatment (36). At the same time, another study by Bao et al. reported that miR-93 suppresses tumorigenesis and enhances chemosensitivity of BRCA by dual targeting E2F1 and CCND1 but did not mention the role of EMT (4). In addition, emerging evidence has shown that cyclinassociated proteins are involved in regulating immune checkpoint proteins. For example, in patients with advanced melanoma, there is a negative correlation between CDK4 copy number gain and the anti-PD1 response, and genetic aberrations of the CDK4 pathway are associated with innate resistance to anti-PD-1 therapy (37). Furthermore, among 32 acral subtype melanoma patients, CCND1 copy number gain was associated with a lack of response to anti-PD-1 therapy (37). Moreover, another mouse tumor model study suggested that cyclin D-CDK4 and the cullin 3-SPOP E3 ligase regulate the expression of PD-L1 protein via proteasome-mediated degradation and that CDK4/6 inhibitor treatment combined with antiPD-1 immunotherapy enhanced tumor regression and markedly improved overall survival rates (38). Similarly, a study on a renal cell carcinoma also showed that cyclin $\mathrm{D}-\mathrm{CDK} 4 / 6$ plays a rate-limiting role in regulating $\mathrm{PD}$ L1 expression (39). Considering the immune-related 
Page 16 of 19

Yang et al. MiR-93-5p regulates tumorigenesis and tumor immunity in BRCA

Table 2 Characteristics and distribution of breast cancer patients in the TCGA-BRCA cohort

\begin{tabular}{|c|c|c|c|c|c|c|}
\hline Clinicopathological features & \multicolumn{3}{|c|}{ Expression of miR-93-5p } & \multicolumn{3}{|c|}{ Survival } \\
\hline Age & & & NS & & & $<0.05$ \\
\hline$<60$ years & 49 & 19 & & 57 & 11 & \\
\hline$>60$ years & 22 & 10 & & 20 & 12 & \\
\hline Present & 34 & 16 & & 33 & 17 & \\
\hline Absent & 37 & 13 & & 44 & 6 & \\
\hline Pathological grade & & & NS & & & NS \\
\hline Grade II & 51 & 17 & & 55 & 13 & \\
\hline $\mathrm{T} 1 / 2$ & 71 & 27 & & 75 & 23 & \\
\hline $\mathrm{T} 3 / 4$ & 0 & 2 & & 2 & 0 & \\
\hline TNM stage & & & $<0.01$ & & & $<0.01$ \\
\hline$|-I|$ & 49 & 11 & & 58 & 9 & \\
\hline III & 22 & 18 & & 19 & 14 & \\
\hline Relapse & & & NS & & & $<0.0001$ \\
\hline Yes & 19 & 9 & & 11 & 17 & \\
\hline No & 52 & 20 & & 66 & 6 & \\
\hline- & 30 & 16 & & 33 & 13 & \\
\hline HER2 & & & NS & & & NS \\
\hline+ & 15 & 4 & & 13 & 6 & \\
\hline - & 56 & 25 & & 64 & 17 & \\
\hline TNBC & & & NS & & & NS \\
\hline Yes & 12 & 8 & & 12 & 8 & \\
\hline No & 49 & 21 & & 65 & 15 & \\
\hline Expression of miR-93-5p & & & - & & & NS \\
\hline Low & - & - & & 55 & 16 & \\
\hline High & - & - & & 22 & 7 & \\
\hline
\end{tabular}

NS, not significant; TNBC, triple negative breast cancer; ER, estrogen receptor; PR, progesterone receptor. 
possibilities of CCND1, our BRCA cell work focused on the correlation of PD-L1 and CCND1 and discovered high expression levels of PD-L1 and CCND1 in MDAMB-231 cells. Simultaneously, analysis of TIMER datasets also predicted that PD-L1 was positively correlated with CCND1. Based on the above data on these two proteins, immunohistochemical staining indicated that PD-L1 and CCND1 were expressed at high levels in BRCA tissues compared to the noncancerous group. We then disrupted expression levels of PD-L1 and CCND1 in MDA-MB-231 cells to determine the interaction between PD-L1 and CCND1, and the results revealed that downregulation of PD-L1 led to diminished expression levels of CCND1, while attenuating the expression of CCND1 augmented expression levels of PD-L1. Hence, we propose the presence of a feedback loop between PD-L1 and CCND1. CCND1 is localized in the cytoplasm and nucleus, and PD$\mathrm{L} 1$ is located in the plasma membrane. When miR-93-5p was knocked down, PD-L1 and CCND1 simultaneously controlled each other, while expression of CCND1 in the nucleus was not disturbed due to its association with CDK4, accumulation on the nuclear membrane, and subsequent transfer into the nucleus. Our study confirmed that miR-93$5 p$ decreases the concentration of CCND1 in the cytoplasm by reducing the formation of CCND 1 and the CDK4 complex. According to the results, miR-93-5p did not affect other cyclin-associated proteins other than CCND1, such as CDK1, CDK4, and cyclin B1.

In conclusion, we confirmed that miR-93-5p is correlated with tumor progression and immune regulation in BRCA and that it directly targets PD-L1 and CCND1 to execute its functions. However, further in vivo studies are required to characterize the miR-93-5p-PD-L1/CCND1-immune cascade, which may provide straightforward prospects for BRCA patients. In short, our research furnishes a rationale for therapy with $m i R-93-5 p$ to overcome immune escape and improve risk stratification in BRCA.

\section{Acknowledgments}

Funding: This work was supported by grants from the National Natural Science Foundation of China (No. 31670895); Key Scientific Research Projects of Higher Education Institutions of Henan Province of China (No. 16A35003); the Department of Science and Technology of Henan Province of China (No. 182102410007); and the Medical Science and Technology Project of Henan Province (No. LHGJ20200333).

\section{Footnote}

Reporting Checklist: The authors have completed the MDAR reporting checklist. Available at https://atm.amegroups. com/article/view/10.21037/atm-22-97/rc

Data Sharing Statement: Available at https://atm.amegroups. com/article/view/10.21037/atm-22-97/dss

Conflicts of Interest: All authors have completed the ICMJE uniform disclosure form (available at https://atm. amegroups.com/article/view/10.21037/atm-22-97/coif). All authors report grants from the National Natural Science Foundation of China (No. 31670895); Key Scientific Research Projects of Higher Education Institutions of Henan Province of China (No. 16A35003); the Department of Science and Technology of Henan Province of China (No. 182102410007); and the Medical Science and Technology Project of Henan Province (No. LHGJ20200333). The authors have no other conflicts of interest to declare.

Etbical Statement: The authors are accountable for all aspects of the work in ensuring that questions related to the accuracy or integrity of any part of the work are appropriately investigated and resolved. All procedures performed in this study involving human participants were in accordance with the Declaration of Helsinki (as revised in 2013). The study was approved by Ethics Committee of the First Affiliated Hospital of Zhengzhou University (No. 2020-KY-449) and informed consent was taken from all the patients.

Open Access Statement: This is an Open Access article distributed in accordance with the Creative Commons Attribution-NonCommercial-NoDerivs 4.0 International License (CC BY-NC-ND 4.0), which permits the noncommercial replication and distribution of the article with the strict proviso that no changes or edits are made and the original work is properly cited (including links to both the formal publication through the relevant DOI and the license). See: https://creativecommons.org/licenses/by-nc-nd/4.0/.

\section{References}

1. Pashayan N, Antoniou AC, Ivanus U, et al. Personalized early detection and prevention of breast cancer: ENVISION consensus statement. Nat Rev Clin Oncol 2020;17:687-705.

2. Harbeck N, Penault-Llorca F, Cortes J, et al. Breast 
cancer. Nat Rev Dis Primers 2019;5:66.

3. Britt KL, Cuzick J, Phillips KA. Key steps for effective breast cancer prevention. Nat Rev Cancer 2020;20:417-36.

4. Bao C, Chen J, Chen D, et al. MiR-93 suppresses tumorigenesis and enhances chemosensitivity of breast cancer via dual targeting E2F1 and CCND1. Cell Death Dis 2020;11:618.

5. Krol J, Loedige I, Filipowicz W. The widespread regulation of microRNA biogenesis, function and decay. Nat Rev Genet 2010;11:597-610.

6. Bartel DP. MicroRNAs: genomics, biogenesis, mechanism, and function. Cell 2004;116:281-97.

7. Calin GA, Croce CM. MicroRNA signatures in human cancers. Nat Rev Cancer 2006;6:857-66.

8. Cortez MA, Anfossi S, Ramapriyan R, et al. Role of miRNAs in immune responses and immunotherapy in cancer. Genes Chromosomes Cancer 2019;58:244-53.

9. Berindan-Neagoe I, Monroig Pdel C, Pasculli B, et al. MicroRNAome genome: a treasure for cancer diagnosis and therapy. CA Cancer J Clin 2014;64:311-36.

10. Beg MS, Brenner AJ, Sachdev J, et al. Phase I study of MRX34, a liposomal miR-34a mimic, administered twice weekly in patients with advanced solid tumors. Invest New Drugs 2017;35:180-8.

11. Wei J, Wang F, Kong LY, et al. miR-124 inhibits STAT3 signaling to enhance $\mathrm{T}$ cell-mediated immune clearance of glioma. Cancer Res 2013;73:3913-26.

12. Xu S, Tao Z, Hai B, et al. miR-424(322) reverses chemoresistance via $\mathrm{T}$-cell immune response activation by blocking the PD-L1 immune checkpoint. Nat Commun 2016;7:11406.

13. Wei J, Nduom EK, Kong LY, et al. MiR-138 exerts antiglioma efficacy by targeting immune checkpoints. Neuro Oncol 2016;18:639-48.

14. Ji Y, Wrzesinski C, Yu Z, et al. miR-155 augments CD8+ T-cell antitumor activity in lymphoreplete hosts by enhancing responsiveness to homeostatic gammac cytokines. Proc Natl Acad Sci U S A 2015;112:476-81.

15. Beatty GL, Gladney WL. Immune escape mechanisms as a guide for cancer immunotherapy. Clin Cancer Res 2015;21:687-92.

16. Grivennikov SI, Greten FR, Karin M. Immunity, inflammation, and cancer. Cell 2010;140:883-99.

17. Nagy Á, Munkácsy G, Győrffy B. Pancancer survival analysis of cancer hallmark genes. Sci Rep 2021;11:6047.

18. Agarwal V, Bell GW, Nam JW, et al. Predicting effective microRNA target sites in mammalian mRNAs. Elife 2015.

19. Sticht C, De La Torre C, Parveen A, et al. miRWalk: An online resource for prediction of microRNA binding sites. PLoS One 2018;13:e0206239.

20. Zhou Y, Zhou B, Pache L, et al. Metascape provides a biologist-oriented resource for the analysis of systemslevel datasets. Nat Commun 2019;10:1523.

21. Chandrashekar DS, Bashel B, Balasubramanya SAH, et al. UALCAN: A Portal for Facilitating Tumor Subgroup Gene Expression and Survival Analyses. Neoplasia 2017;19:649-58.

22. Li JH, Liu S, Zhou H, et al. starBase v2.0: decoding miRNA-ceRNA, miRNA-ncRNA and protein-RNA interaction networks from large-scale CLIP-Seq data. Nucleic Acids Res 2014;42:D92-7.

23. Li T, Fu J, Zeng Z, et al. TIMER2.0 for analysis of tumor-infiltrating immune cells. Nucleic Acids Res 2020;48:W509-14.

24. Lu J, Getz G, Miska EA, et al. MicroRNA expression profiles classify human cancers. Nature 2005;435:834-8.

25. Mattie MD, Benz CC, Bowers J, et al. Optimized highthroughput microRNA expression profiling provides novel biomarker assessment of clinical prostate and breast cancer biopsies. Mol Cancer 2006;5:24.

26. Fridrichova I, Zmetakova I. MicroRNAs Contribute to Breast Cancer Invasiveness. Cells 2019;8:1361.

27. Hay M, Thomas DW, Craighead JL, et al. Clinical development success rates for investigational drugs. Nat Biotechnol 2014;32:40-51.

28. Li L, Zhao J, Huang S, et al. MiR-93-5p promotes gastric cancer-cell progression via inactivation of the Hippo signaling pathway. Gene 2018;641:240-7.

29. Ma DH, Li BS, Liu JJ, et al. miR-93-5p/IFNAR1 axis promotes gastric cancer metastasis through activating the STAT3 signaling pathway. Cancer Lett 2017;408:23-32.

30. Sun XY, Han XM, Zhao XL, et al. MiR-93-5p promotes cervical cancer progression by targeting THBS2/ MMPS signal pathway. Eur Rev Med Pharmacol Sci 2019;23:5113-21.

31. Chen S, Chen X, Sun KX, et al. MicroRNA-93 Promotes Epithelial-Mesenchymal Transition of Endometrial Carcinoma Cells. PLoS One 2016;11:e0165776.

32. Wu H, Liu L, Zhu JM. MiR-93-5p inhibited proliferation and metastasis of glioma cells by targeting MMP2. Eur Rev Med Pharmacol Sci 2019;23:9517-24.

33. Chen YL, Wang GX, Lin BA, et al. MicroRNA-93$5 \mathrm{p}$ expression in tumor tissue and its tumor suppressor function via targeting programmed death ligand-1 in colorectal cancer. Cell Biol Int 2020;44:1224-36.

34. Shyamasundar S, Lim JP, Bay BH. miR-93 inhibits 
the invasive potential of triple-negative breast cancer cells in vitro via protein kinase WNK1. Int J Oncol 2016;49:2629-36.

35. Xiang Y, Liao XH, Yu CX, et al. MiR-93-5p inhibits the EMT of breast cancer cells via targeting MKL-1 and STAT3. Exp Cell Res 2017;357:135-44.

36. Tobin NP, Sims AH, Lundgren KL, et al. Cyclin D1, Id1 and EMT in breast cancer. BMC Cancer 2011;11:417.

37. Yu J, Yan J, Guo Q, et al. Genetic Aberrations in the CDK4 Pathway Are Associated with Innate Resistance to

Cite this article as: Yang M, Xiao R, Wang X, Xiong Y, Duan Z, Li D, Kan Q. MiR-93-5p regulates tumorigenesis and tumor immunity by targeting PD-L1/CCND1 in breast cancer. Ann Transl Med 2022;10(4):203. doi: 10.21037/atm-22-97
PD-1 Blockade in Chinese Patients with Non-Cutaneous Melanoma. Clin Cancer Res 2019;25:6511-23.

38. Zhang J, Bu X, Wang H, et al. Cyclin D-CDK4 kinase destabilizes PD-L1 via cullin 3-SPOP to control cancer immune surveillance. Nature 2018;553:91-5.

39. Qin Z, Hu H, Sun W, et al. miR-224-5p Contained in Urinary Extracellular Vesicles Regulates PD-L1 Expression by Inhibiting Cyclin D1 in Renal Cell Carcinoma Cells. Cancers (Basel) 2021;13:618. 